\title{
Structure Learning of $\mathrm{H}$-Colorings
}

\author{
ANTONIO BLANCA and ZONGCHEN CHEN, Georgia Institute of Technology \\ DANIEL ŠTEFANKOVIČ, University of Rochester \\ ERIC VIGODA, Georgia Institute of Technology
}

\begin{abstract}
We study the following structure learning problem for $H$-colorings. For a fixed (and known) constraint graph $H$ with $q$ colors, given access to uniformly random $H$-colorings of an unknown graph $G=(V, E)$, how many samples are required to learn the edges of $G$ ? We give a characterization of the constraint graphs $H$ for which the problem is identifiable for every $G$ and show that there are identifiable constraint graphs for which one cannot hope to learn every graph $G$ efficiently. We provide refined results for the case of proper vertex $q$ colorings of graphs of maximum degree $d$. In particular, we prove that in the tree uniqueness region (i.e., when $q>d)$, the problem is identifiable and we can learn $G$ in poly $(d, q) \times O\left(n^{2} \log n\right)$ time. In the tree nonuniqueness region (i.e., when $q \leq d$ ), we show that the problem is not identifiable and thus $G$ cannot be learned. Moreover, when $q \leq d-\sqrt{d}+\Theta(1)$, we establish that even learning an equivalent graph (any graph with the same set of $H$-colorings) is computationally hard-sample complexity is exponential in $n$ in the worst case. We further explore the connection between the efficiency/hardness of the structure learning problem and the uniqueness/non-uniqueness phase transition for general $H$-colorings and prove that under a wellknown uniqueness condition in statistical physics, we can learn $G$ in poly $(d, q) \times O\left(n^{2} \log n\right)$ time.
\end{abstract}

CCS Concepts: • Mathematics of computing $\rightarrow$ Probabilistic inference problems; • Theory of computation $\rightarrow$ Machine learning theory; Design and analysis of algorithms;

Additional Key Words and Phrases: Structure learning, identifiability, Markov random fields, H-colorings, spin systems

\section{ACM Reference format:}

Antonio Blanca, Zongchen Chen, Daniel Štefankovič, and Eric Vigoda. 2020. Structure Learning of $H$ Colorings. ACM Trans. Algorithms 16, 3, Article 36 (May 2020), 28 pages.

https://doi.org/10.1145/3382207

\section{INTRODUCTION}

Structure learning is a general framework for supervised learning where instead of learning labels or real numbers as in classification or regression, the task is to learn a more complex structure, such as a graph. We study structure learning for Markov random fields (undirected graphical models) where the goal is to recover the underlying graph from random samples; this problem has found important applications in diverse fields (e.g., [21, 30, 36, 39, 41, 42]).

Authors' addresses: A. Blanca, Z. Chen, and E. Vigoda, Georgia Institute of Technology, Klaus Advanced Computing Building, North Ave NW, Atlanta, GA 30332; emails: ablanca@cc.gatech.edu, \{chenzongchen, vigoda\}@gatech.edu; D. Štefankovič, University of Rochester, Department of Computer Science, 2315 Wegmans Hall, Rochester NY, 14627; email: stefanko@cs.rochester.edu.

Permission to make digital or hard copies of all or part of this work for personal or classroom use is granted without fee provided that copies are not made or distributed for profit or commercial advantage and that copies bear this notice and the full citation on the first page. Copyrights for components of this work owned by others than the author(s) must be honored. Abstracting with credit is permitted. To copy otherwise, or republish, to post on servers or to redistribute to lists, requires prior specific permission and/or a fee. Request permissions from permissions@acm.org.

(C) 2020 Copyright held by the owner/author(s). Publication rights licensed to ACM.

1549-6325/2020/05-ART36 \$15.00

https://doi.org/10.1145/3382207 
Our goal in this work is to understand when structure learning for Markov random fields is possible in polynomial time. We mostly focus on the task of exact recovery, where a learning algorithm is said to succeed only when it outputs exactly the hidden graph. In applications, exact recovery of graphical models is often of interest, since the true graph structure contains valuable information about the dependencies in the model. Consequently, it has been very well studied (e.g., see [2, 4, 5, 8, 11-13, 27, 31, 33, 34, 40, 46, 47]). Although the typical setting in these works is soft-constraint models, our focus here is models with hard constraints. Specifically, we consider the structure learning problem and the closely related question of statistical identifiability in the general setting of $H$-colorings. $H$-colorings are studied in several other contexts, with the associated computational problems receiving substantial attention; notably, the decision problem [10, $28,29,43]$ and the exact and approximate counting/sampling problems [17, 19, 23, 26] have been considered extensively.

Given an undirected, connected constraint graph $H=(V(H), E(H))$, with vertices $V(H)=$ $\{1, \ldots, q\}$ referred to as colors (or spins), an $H$-coloring of a graph $G=(V, E)$ is an assignment of colors $\{1, \ldots, q\}$ to the vertices of $G$ such that adjacent vertices of $G$ receive adjacent colors in $H$. In other words, an $H$-coloring $\sigma$ is a mapping $\sigma: V \rightarrow V(H)$ such that if $\{v, w\} \in E$, then $\{\sigma(v), \sigma(w)\} \in E(H)$. If such an assignment is possible, we say that $G$ is $H$-colorable. The constraint graph $H$ is allowed to have self-loops, but not parallel edges, and every $\{i, j\}$ such that $\{i, j\} \notin E(H)$ is called a hard constraint.

Two canonical examples of $H$-colorings are proper colorings and independent sets. The former corresponds to the case where $H$ is the complete graph $K_{q}$ on $q$ vertices with no selfloops and the latter to the constraint graph $H$ with two vertices $V(H)=\{0,1\}$ and two edges $E(H)=\{\{0,0\},\{0,1\}\}$. Spin systems without hard constraints (i.e., soft-constraint systems) correspond to $H=K_{q}^{+}$, where $K_{q}^{+}$denotes the complete graph with a self-loop at every vertex. In this case, all $q^{|V|}$ labelings of $G$ are valid $H$-colorings.

We consider structure learning and statistical identifiability for $H$-colorings with at least one hard constraint-that is, $H \neq K_{q}^{+}$. Note that the missing edge could be a self-loop.

For an $H$-colorable graph $G$, let $\Omega_{G}^{H}$ be the set of all possible $H$-colorings of $G$ and let $\pi_{G}^{H}$ denote the uniform measure over $\Omega_{G}^{H}$. Typically, the constraint graph $H$ will be fixed, and thus we will drop the dependence on $H$ in our notation. Some of our results extend to the more general setting of a weighted constraint graph $H$ and a weighted graph $G$, where $\pi_{G}^{H}$ is the corresponding Gibbs distribution; see Section 6.1 for a precise definition.

We first address the statistical identifiability problem for general $H$-colorings, where the goal is to characterize the cases when every graph is learnable with an infinite number of samples.

Definition 1.1. A constraint graph $H$ is said to be identifiable with respect to a family of $H$ colorable graphs $\mathcal{G}$ if for any two distinct graphs $G_{1}, G_{2} \in \mathcal{G}$ we have $\pi_{G_{1}} \neq \pi_{G_{2}}$ (or equivalently $\Omega_{G_{1}} \neq \Omega_{G_{2}}$ ). In particular, when $\mathcal{G}$ is the set of all finite $H$-colorable graphs, we say that $H$ is identifiable.

To characterize identifiability, we introduce a supergraph $G_{i j}$ of $H$ for $\{i, j\} \in E(H)$. This supergraph will not be used as a constraint graph, but rather we will consider the $H$-colorings of $G_{i j}$ in our characterization theorem. Consider an edge $\{i, j\} \in E(H)$. We construct $G_{i j}$ by starting from $H$ and duplicating the colors $i$ and $j$. These new copies, denoted $i^{\prime}$ and $j^{\prime}$, have the same neighbors as the original colors $i$ and $j$, respectively, except for the one edge $\left\{i^{\prime}, j^{\prime}\right\}$, which is not included; see Figure 1 for an illustration of this supergraph and Definition 2.2 for a formal definition.

For a constraint graph $H$, we say that a pair of colors $i, j \in V(H)$ is compatible (respectively, incompatible) if $\{i, j\} \in E(H)$ (respectively, $\{i, j\} \notin E(H)$ ). Our characterization theorem considers whether in every $H$-coloring of $G_{i j}$ the new vertices $i^{\prime}, j^{\prime}$ receive compatible colors. 


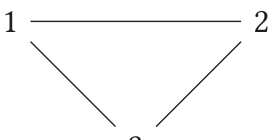

3

$H$

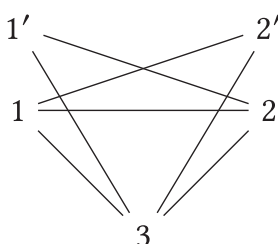

3

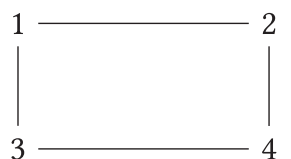

$H^{\prime}$

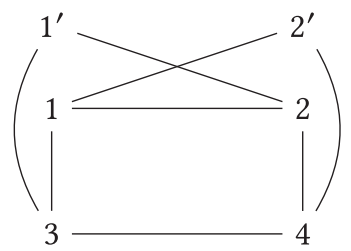

$G_{12}^{\prime}$

Fig. 1. Two constraint graphs $H$ and $H^{\prime}$ with corresponding supergraphs $G_{12}$ and $G_{12}^{\prime}$.

THeOREM 1.2. Let $H \neq K_{q}^{+}$be an arbitrary constraint graph. If $H$ has at least one self-loop, then $H$ is identifiable. Otherwise, $H$ is identifiable if and only if for each $\{i, j\} \in E(H)$ there exists an $H$ coloring $\sigma$ of $G_{i j}$ such that $\sigma\left(i^{\prime}\right)$ and $\sigma\left(j^{\prime}\right)$ are incompatible colors in $H$.

The crucial role of the graph $G_{i j}$ for identifiability is elucidated in Section 2. We are also able to extend this characterization theorem to the case of weighted $\mathrm{H}$-colorings; see Theorem 6.7 in Section 6.4 for a precise statement.

For structure learning, our goal is to efficiently learn the graph $G$ from samples drawn independently from $\pi_{G}$. More formally, let $H$ be a fixed constraint graph and let $\mathcal{G}$ be a family of $H$-colorable graphs. Suppose that we are given $L$ samples $\sigma^{(1)}, \sigma^{(2)}, \ldots, \sigma^{(L)}$ drawn independently from the distribution $\pi_{G}^{H}$ where $G \in \mathcal{G}$. A structure learning algorithm for the constraint graph $H$ and the graph family $\mathcal{G}$ takes as input the sample sequence $\sigma^{(1)}, \sigma^{(2)}, \ldots, \sigma^{(L)}$ and outputs an estimator $\hat{G} \in \mathcal{G}$ such that $\operatorname{Pr}[G=\hat{G}] \geq 1-\varepsilon$ where $\varepsilon>0$ is a prescribed error (failure probability).

As mentioned earlier, structure learning has been well studied for soft-constraint models $(H=$ $K_{q}^{+}$). For a system with maximum interaction strength $\beta$ on an unknown $n$-vertex graph $G$ of maximum degree $d$, Klivans and Meka [33] provide a structure learning algorithm that achieves nearly optimal running time of $O\left(n^{2} \log n\right) \times \exp (O(\beta d))$; see earlier related work [4, 27, 47]. All of these results concern soft-constraint models and do not apply to the setting of hard-constraint systems. We shall see that although the algorithm in Klivans and Meka [33] achieves (optimal) single exponential dependence on $d$ for general soft-constraint systems, the structure learning problem for hard-constraint systems is quite different. Indeed, some hard-constraint systems are not statistically identifiable (and thus the unknown graph $G$ cannot be learned); others allow very efficient structure learning algorithms with poly $(n, d, q)$ running time; whereas also in others, any structure learning algorithm requires exponentially (in $n$ ) many samples.

For hard-constraint systems, the structure learning problem was previously studied by Bresler et al. [6] for independent sets (more generally, for the hard-core model where the independent sets are weighted by their size and a model parameter $\lambda>0$ ). They achieve nearly optimal running time of $O\left(n^{2} \log n\right) \times \exp (O(d \lambda))$. For our positive results, we generalize the structure learning algorithm in Bresler et al. [6].

Let $\mathcal{G}(n, d)$ be the family of $n$-vertex graphs of maximum degree at most $d$. Our next result shows that for some identifiable (with respect to $\mathcal{G}(n, d)$ ) constraint graphs, one cannot hope to learn the underlying graph $G$ efficiently. As mentioned, this is not the case for soft-constraint models, where one can always learn $G$ in time $O\left(n^{2} \log n\right) \times \exp (O(d \beta))$ [33].

THEOREM 1.3. There exists an identifiable constraint graph $H$ and a constant $c>0$ such that for all $n \geq 8$, any structure learning algorithm for $H$ and the graph family $\mathcal{G}(n, 7)$ that succeeds with probability at least $\exp (-c n)$ requires at least $\exp (\mathrm{cn})$ samples. 
Although this theorem shows that there is no efficient learning algorithm for all identifiable models, for some relevant models structure learning can be done efficiently.

We focus first on the case of proper $q$-colorings where $H=K_{q}$. In general, the proper colorings problem is not identifiable. However, if we consider identifiability with respect to the graph family $\mathcal{G}(n, d)$, we get a richer picture. We prove that when $q>d$, the $q$-colorings problem is identifiable, whereas when $q \leq d$, it is not. This is a natural threshold due to the efficiency of the simple greedy algorithm for constructing a $q$-coloring. In addition, it coincides with the statistical physics uniqueness/non-uniqueness phase transition of the Gibbs distribution on infinite $d$-regular trees (see $[9,32])$, which is known to be closely connected to the efficiency/hardness of other fundamental computational problems, such as sampling and counting (e.g., see [24, 35, 44, 45, 49]).

In the identifiable region $q>d$, we present an efficient structure learning algorithm with $O\left(n^{2} \log n\right) \times$ poly $(d, q)$ running time. When $q \leq d$, where we cannot hope to learn the hidden graph $G$ since there are pairs of graphs with the same set of $H$-colorings, we may be interested in a learning algorithm that outputs a graph $G^{\prime}$ that is equivalent to the unknown graph $G$, in the sense that $\Omega_{G^{\prime}}=\Omega_{G}$. We say that an algorithm is an equivalent-structure learning algorithm for a fixed constraint graph $H$ and a graph family $\mathcal{G}$ if for every $G \in \mathcal{G}$, with probability at least $1-\varepsilon$, the algorithm outputs $G^{\prime} \in \mathcal{G}$ such that $\Omega_{G}=\Omega_{G^{\prime}}$.

In the $q$-coloring setting, it turns out that when $q \leq d-\sqrt{d}+\Theta(1)$, there is a family of exponentially many $n$-vertex graphs with different sets of $q$-colorings that only differ on an exponentially small (in $n$ ) fraction of their $q$-colorings. Consequently, any equivalent-structure learning algorithm requires exponential many samples to distinguish among these graphs. The $q \leq d-\sqrt{d}+\Theta(1)$ threshold is quite curious since it is exactly the same as the one for polynomialtime/NP-completeness for the decision problem [22, 38], and it was recently shown [3] that it also aligns with the polynomial-time/\#BIS-hardness transition for the identity testing problem for proper colorings. The graph used in our proof here is inspired by the graphs used in other works $[22,38]$.

Our results for statistical identifiability, structure learning, and equivalent-structure learning for proper $q$-colorings are stated in the following theorem.

TheOREM 1.4. Consider the q-colorings problem $H=K_{q}$. The following hold for all d:

(1) Efficient learning for $q>d$ : For all $q>d, n \geq 1$, and any $G \in \mathcal{G}(n, d)$, there is a structure learning algorithm that given $L=O\left(q d^{3} \log \left(\frac{n^{2}}{\varepsilon}\right)\right)$ independent samples from $\pi_{G}$ outputs $G$ with probability at least $1-\varepsilon$ and has running time $O\left(L n^{2}\right)$.

(2) Non-identifiability for $q \leq d$ : For all $q \leq d$ and $n \geq q+2$, there exist $q$-colorable graphs $G_{1}, G_{2} \in \mathcal{G}(n, d)$ such that $G_{1} \neq G_{2}$ and $\pi_{G_{1}}=\pi_{G_{2}}$.

(3) Lower bound for $q \leq d-\sqrt{d}+\Theta(1)$ : For all $d \geq q+\lceil\sqrt{q}\rceil-1$, there exists a constant $c>0$ such that any equivalent-structure learning algorithm for the family of graphs $\mathcal{G}(n, d)$ that succeeds with probability at least $\exp (-c n)$ requires at least $\exp (\mathrm{cn})$ samples, provided $n$ is sufficiently large.

(4) Lower bound for $q \leq d$ : For all $3 \leq q \leq d$ and $n \geq d+2$, any equivalent-structure learning algorithm for $\mathcal{G}(n, d)$ that succeeds with probability at least $1 / 2$ requires $\exp (\Omega(d-q))$ samples.

We remark that part (3) of this theorem provides an exponential lower bound for equivalentstructure learning in the non-identifiable regime, whereas Theorem 1.3 provides a similar lower bound but for structure learning for an identifiable model.

Our results for the structure learning problem in the setting of $q$-colorings suggest an intimate connection between the efficiency/hardness of the learning problem and the uniqueness/ 
non-uniqueness phase transition of the Gibbs distribution. We further explore this connection for general $H$-colorings.

A well-known sufficient condition for uniqueness on general graphs concerns the maximum influence of a site. Specifically, if $\tau, \tau_{w}$ are pairs of spin assignments of a graph $G=(V, E)$ that differ only at vertex $w$, we can define the so-called influence matrix $R$ whose entry $R_{v, w}$ corresponds to the maximum (over the pairs $\tau, \tau_{w}$ ) of the difference in total variation distance of the marginal distribution at vertex $v$ conditional on the spin assignment $\tau(V \backslash v)$ versus $\tau_{w}(V \backslash v)$; see Definition 4.1. If the maximum column sum of $R$ is strictly less than 1 (i.e., $\alpha:=\max _{w \in V} \sum_{v \in \partial w} R_{v, w}<1$ ), then the influence of any vertex on its neighborhood is less than 1. (Observe that $R_{v, w}=0$ for nonadjacent pairs $v, w$.) Dobrushin and Shlosman [16] were the first to derive uniqueness from this type of condition; see Remark 4.2 for some additional background on the $\alpha<1$ condition. We show that we can learn the underlying $n$-vertex graph in $\operatorname{poly}(n, q)$ time when $\alpha<1$.

Theorem 1.5. Let $H \neq K_{q}^{+}$be an arbitrary constraint graph. Suppose $G$ is such that the uniqueness condition $\alpha<1$ is satisfied for $\pi_{G}$. Then, there is a structure learning algorithm that given $L=O\left(q^{2} \log \left(\frac{n^{2}}{\varepsilon}\right)\right)$ independent samples from $\pi_{G}$ outputs the graph $G$ with probability at least $1-\varepsilon$ and has running time $O\left(\operatorname{Ln}^{2}\right)$.

Under a much weaker (i.e., easier to satisfy) condition, we give a structure learning algorithm with running time exponential in the maximum degree $d$ of the graph $G$. Our algorithm works for all permissive systems and generalizes to the weighted setting. The precise definition, as well as the running time and sample complexity of our algorithm, are provided in Section 6; see Definition 6.1 and Theorem 6.2.

Finally, we mention that the corresponding approximation problem of finding a graph $G^{\prime}$ such that $\pi_{G^{\prime}}$ and $\pi_{G}$ are close in some notion of distance, which is not yet well understood for softconstraint systems [1,7], is apparently much simpler for hard-constraint systems. In Section 5 , we provide an algorithm for approximate structure learning for all hard-constraint systems with polynomial running time and sample complexity (see Theorem 5.1).

The rest of the article is organized as follows. In Section 2, we prove our characterization theorem (Theorem 1.2) and our learning lower bound for identifiable models (Theorem 1.3). In Section 3, we prove our results for colorings (Theorem 1.4). In particular, in Section 3.1, we introduce a general structure learning algorithm STRUCTLEARN-H, which will be the basis of all of our algorithmic result. Our poly $(n, d, q)$-time algorithm in the uniqueness regime (Theorem 1.5) is established in Section 4. In Section 5, we consider the approximation problem of learning a graph $G$ such that $\pi_{G}$ is close in total variation distance to the true distribution. Finally, the case of weighted $H$ and $G$ is considered in Section 6.

\section{IDENTIFIABILITY}

As discussed in Section 1, given a constraint graph $H$, it is possible that $\pi_{G_{1}}=\pi_{G_{2}}$ for two distinct $H$-colorable graphs $G_{1}$ and $G_{2}$-that is, the structure learning problem is not identifiable. In this section, we prove Theorem 1.2 from Section 1 that characterizes the identifiable constraint graphs.

Let $\mathcal{G}$ be a family of $H$-colorable graphs. Recall that a constraint graph $H$ is identifiable with respect to $\mathcal{G}$ if for any two distinct graphs $G_{1}, G_{2} \in \mathcal{G}$ we have $\pi_{G_{1}} \neq \pi_{G_{2}}$ or equivalently $\Omega_{G_{1}} \neq \Omega_{G_{2}}$. In particular, when $\mathcal{G}$ is the set of all finite $H$-colorable graphs, we say that $H$ is identifiable; see Definition 1.1. Before proving Theorem 1.2, we provide the following useful alternative definition of identifiability.

LEMMA 2.1. A constraint graph $H \neq K_{q}^{+}$is identifiable if and only if for any $H$-colorable graph $G=(V, E)$ and any two non-adjacent vertices $u, v \in V$ there is an $H$-coloring of $G$ where $u$ and $v$ are assigned incompatible colors. 
Proof. For the forward direction, we prove the contrapositive. Let $G=(V, E)$ be an $H$-colorable graph and suppose there exists two non-adjacent vertices $u, v \in V$ such that in every $H$-coloring $\sigma$ of $G$ these vertices receive compatible colors. Then, the graph $G$ and the graph $G^{\prime}=(V, E \cup\{u, v\})$ have the same set of $H$-colorings. Hence, $\pi_{G}=\pi_{G^{\prime}}$ and thus $H$ is not identifiable.

For the reverse direction, suppose that for every $H$-colorable graph $G=(V, E)$ and every pair of non-adjacent vertices $u, v \in V$ there exists an $H$-coloring of $G$ such that $u$ and $v$ are assigned incompatible colors. Suppose also that for a pair of $H$-colorable graphs $G_{1}=\left(V, E_{1}\right)$ and $G_{2}=$ $\left(V, E_{2}\right)$, we have $\pi_{G_{1}}=\pi_{G_{2}}$ (or equivalently that $\Omega_{G_{1}}=\Omega_{G_{2}}$ ). We show that $G_{1}=G_{2}$. First consider $\{u, v\} \notin E_{1}$. Then, there exists an $H$-coloring $\tau \in \Omega_{G_{1}}$ where $u$ and $v$ receive incompatible colors. Since also $\tau \in \Omega_{G_{2}},\{u, v\} \notin E_{2}$. Similarly, if $\{u, v\} \notin E_{2}$, then $\{u, v\} \notin E_{1}$. Thus, $G_{1}=G_{2}$ and thus $H$ is identifiable.

To characterize identifiability of $H$-colorings, we previously introduced the supergraph $G_{i j}$ of $H$ obtained by duplicating the colors $i$ and $j$. The new copies of $i$ and $j$, denoted $i^{\prime}$ and $j^{\prime}$, have the same neighbors as $i$ and $j$, respectively, except for the one edge $\left\{i^{\prime}, j^{\prime}\right\}$ that is not present in $G_{i j}$-see Figure 1 for examples of the graph $G_{i j}$. We provide next the formal definition of $G_{i j}$.

Definition 2.2. Let $H=(V(H), E(H))$ be an arbitrary constraint graph with no self-loops. For each $\{i, j\} \in E(H)$, we define the graph $G_{i j}=\left(V\left(G_{i j}\right), E\left(G_{i j}\right)\right)$ as follows:

(1) $V\left(G_{i j}\right)=V(H) \cup\left\{i^{\prime}, j^{\prime}\right\}$, where $i^{\prime}$ and $j^{\prime}$ are two new colors;

(2) If $\{a, b\} \in E(H)$, then the edge $\{a, b\}$ is also in $E\left(G_{i j}\right)$;

(3) For each $k \in V\left(G_{i j}\right) \backslash\left\{i^{\prime}, j^{\prime}\right\}$, the edge $\left\{i^{\prime}, k\right\}$ is in $G_{i j}$ if and only if the edge $\{i, k\}$ is in $H$, and similarly $\left\{j^{\prime}, k\right\} \in E\left(G_{i j}\right)$ if and only if $\{j, k\} \in E(H)$.

The intuition for the role of the graph $G_{i j}$ in our characterization theorem is that if we added the edge $\left\{i^{\prime}, j^{\prime}\right\}$ to $G_{i j}$ to form the graph $G_{i j}^{\prime}=G_{i j} \cup\left\{i^{\prime}, j^{\prime}\right\}$, then in every $H$-coloring of $G_{i j}^{\prime}$ the vertices $i^{\prime}, j^{\prime}$ receive compatible colors. If the same property holds for $G_{i j}$-that is, in every $H^{-}$ coloring of $G_{i j}$ the vertices $i^{\prime}, j^{\prime}$ receive compatible colors-then the edge $\left\{i^{\prime}, j^{\prime}\right\}$ plays no role and the pair of graphs $G_{i j}$ and $G_{i j}^{\prime}$ have the same set of $H$-colorings. In other words, $G_{i j}$ and $G_{i j}^{\prime}$ are indistinguishable, and thus $H$ is not identifiable.

Our characterization of identifiability (i.e., Theorem 1.2 from Section 1) is established by the next two lemmas. Lemma 2.3 deals with the case of constraint graphs with at least one self-loop, whereas Lemma 2.4 considers constraint graphs with no self-loops.

Lemma 2.3. If $H \neq K_{q}^{+}$has at least one self-loop, then $H$ is identifiable.

Proof. The proof is divided into two cases corresponding to whether all vertices of $H$ have self-loops or not.

Case 1: At least one but not all vertices of $H$ have self-loops. Let $U$ be the set of vertices that have self-loops, and let $W=V(H) \backslash U$ be the set of vertices that do not. By assumption, both $U$ and $W$ are not empty. Moreover, $U$ and $W$ are connected because by assumption $H$ is connected. Thus, there exist $i \in U$ and $j \in W$ such that $\{i, i\},\{i, j\} \in E(H)$ and $\{j, j\} \notin E(H)$. We use this gadget to show that for any $H$-colorable graph $G=(V, E)$ and any two non-adjacent vertices $u, v \in V$ of $G$, there exists an $H$-coloring $\sigma$ of $G$ where $u$ and $v$ are assigned incompatible colors. Then, by Lemma 2.1, $H$ is identifiable. The $H$-coloring $\sigma$ is defined as follows: $\sigma(w)=i$ for all $w \neq u, v$ and $\sigma(u)=\sigma(v)=j$. Since $\{i, i\},\{i, j\} \in E(H), \sigma$ is a valid $H$-coloring of $G$. Moreover, since $\{j, j\} \notin E(H), u$ and $v$ receive incompatible colors and the result follows.

Case 2: All vertices of $H$ have self-loops. Observe first that if $H$ is connected, $H \neq K_{q}^{+}$and every vertex in $H$ has a self-loop, then there exist $i, j, k \in V(H)$ such that $\{i, j\},\{j, k\},\{i, i\},\{j, j\},\{k, k\} \in$ $E(H)$, and $\{i, k\} \notin E(H)$. We use this gadget to show for any $H$-colorable graph $G=(V, E)$ and any 


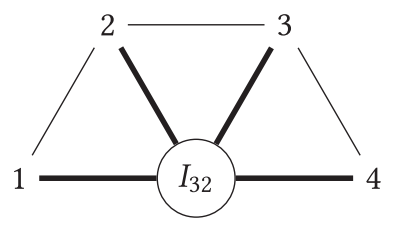

(a)

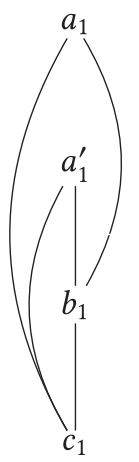

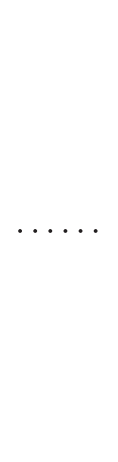

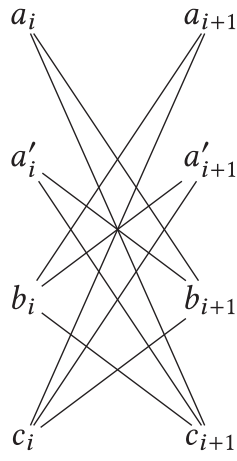

(b)

Fig. 2. (a) The constraint graph $F$; each thick edge corresponds to 32 edges, one incident to each vertex of $I_{32}$. (b) The graph $G_{m}$ showing the edges in $E_{i, i+1}$ and the connections between the vertices $a_{1}, a_{1}^{\prime}, b_{1}, c_{1}$.

pair of non-adjacent vertices $u, v \in V$ there is an $H$-coloring $\sigma$ of $G$ such that $(\sigma(u), \sigma(v)) \notin E(H)$. Lemma 2.1 then implies that $H$ is identifiable. The $H$-coloring $\sigma$ is given by $\sigma(w)=j$ for all $w \neq u, v$, $\sigma(u)=i$ and $\sigma(v)=k$. Since color $j$ is compatible with colors $i, j$, and $k$ in $H, \sigma$ is a valid $H$-coloring of $G$. Moreover, $u$ and $v$ receive the incompatible colors $i$ and $k$ and so the result follows.

LEMMA 2.4. If $H \neq K_{q}^{+}$has no self-loops, $H$ is identifiable if and only if for each $\{i, j\} \in E(H)$ there exists an $H$-coloring of $G_{i j}$ where $i^{\prime}$ and $j^{\prime}$ receive incompatible colors.

Proof. Assume first that $H \neq K_{q}^{+}$is identifiable and has no self-loops. For every $\{i, j\} \in E(H)$, $G_{i j}$ is clearly $H$-colorable (simply assign color $k \in V(H)$ to the corresponding vertex in $G_{i j}$, color $i$ to $i^{\prime}$ and color $j$ to $\left.j^{\prime}\right)$. Hence, Lemma 2.1 implies that there exists an $H$-coloring of $G_{i j}$ where $i^{\prime}$ and $j^{\prime}$ receive incompatible colors. This proves the forward direction of the lemma.

For the reverse direction, suppose that for every $\{i, j\} \in E(H)$ there exists an $H$-coloring of $G_{i j}$ where $i^{\prime}$ and $j^{\prime}$ are assigned incompatible colors. Let $G=(V, E)$ be an arbitrary $H$-colorable graph. We show that for every pair of non-adjacent vertices $u, v \in V$ in $G$ there exists an $H$-coloring of $G$ where $u$ and $v$ receive incompatible colors. It then follows from Lemma 2.1 that $H$ is identifiable.

Let $\sigma$ be an $H$-coloring of $G$, and let us assume that $\sigma(u)$ and $\sigma(v)$ are compatible colors. (If $\sigma(u)$ and $\sigma(v)$ are incompatible colors in $H$, there is nothing to prove.) We use $\sigma$ to construct an $H^{-}$ coloring $\sigma^{\prime}$ where $u$ and $v$ receive incompatible colors. Let $a=\sigma(u)$ and $b=\sigma(v)$. By assumption, there exists an $H$-coloring $\tau$ of $G_{a b}$ where the corresponding copies of $a$ and $b, a^{\prime}$ and $b^{\prime}$, receive incompatible colors. Define the $H$-coloring $\sigma^{\prime}$ of $G$ as follows:

$$
\sigma^{\prime}(w)=\tau(\sigma(w)), \quad \forall w \neq u, v ; \quad \sigma^{\prime}(u)=\tau\left(a^{\prime}\right) ; \quad \sigma^{\prime}(v)=\tau\left(b^{\prime}\right) .
$$

It is straightforward to check that $\sigma^{\prime}$ is a proper $H$-coloring of $G$. Since $u$ and $v$ receive incompatible colors in $\sigma^{\prime}$ (i.e., $\tau\left(a^{\prime}\right)$ and $\tau\left(b^{\prime}\right)$ ), the proof is complete.

\subsection{Learning Lower Bounds for Identifiable Models}

In this section, we prove Theorem 1.3 from Section 1. In particular, we provide a constraint graph $F$ and a family of $F$-colorable $n$-vertex graphs of maximum degree 7 such that the number of samples from $\pi_{G}^{F}$ required to learn any graph in this family, even with success probability $\exp (-O(n))$, is exponential in $n$.

We define the constraint graph $F=(V(F), E(F))$ first, which consists of an independent set of size 32 , denoted $I_{32}$, and four additional vertices $\{1,2,3,4\}$. Every vertex in the independent set $I_{32}$ is connected to these four vertices and also $\{1,2\},\{2,3\},\{3,4\} \in E(F)$; see Figure 2(a). 
LEMMA 2.5. F is identifiable.

Proof. Let $G=(V, E)$ be an $F$-colorable graph. Since $F$ is tripartite with a unique tripartition $\left\{\{1,3\},\{2,4\}, I_{32}\right\}$, then so is $G$. Let $\left\{V_{1}, V_{2}, V_{3}\right\}$ be a tripartition of $G$, and let $u, v$ be any two nonadjacent vertices of $G$. We show that there is always an $F$-coloring of $G$ where $u$ and $v$ receive incompatible colors. The result then follows from Lemma 2.1.

If $u$ and $v$ belong to the same $V_{i}$, then by coloring all vertices $V_{1}$ with color 1 , all vertices of $V_{2}$ with color 2 , and all vertices in $V_{3}$ with any color $c$ from $I_{32}$, we have a coloring of $G$ where $u$ and $v$ receive the same color. Since $F$ has no self-loops, $u$ and $v$ are assigned incompatible colors.

If $u$ and $v$ belong to different $V_{i}$ 's, suppose without loss of generality that $u \in V_{1}$ and $v \in V_{2}$. Consider the following $F$-coloring $\sigma$ of $G$ where $c$ is any color from $I_{32}$ :

$$
\sigma(w)= \begin{cases}1 & \text { if } w=u \\ 4 & \text { if } w=v \\ 3 & \text { if } w \in V_{1} \backslash\{u\} \\ 2 & \text { if } w \in V_{2} \backslash\{v\} \\ c & \text { if } w \in V_{3}\end{cases}
$$

In $\sigma, u$ and $v$ receive the incompatible colors 1 and 4 . Thus, we have shown that it is always possible to color non-adjacent vertices of $G$ with incompatible colors and the result follows from Lemma 2.1.

Next we define a family $\mathcal{G}_{n}$ of $n$-vertex graphs of maximum degree 7 such that every graph in the family has almost the same set of $F$-colorings. Every graph in $\mathcal{G}_{n}$ will be a supergraph of the graph $G_{m}=\left(V_{m}, E_{m}\right)$, whose vertex set is given by

$$
V_{m}=\left\{a_{i}, a_{i}^{\prime}, b_{i}, c_{i}: 1 \leq i \leq m\right\}
$$

with $m=n / 4$. (For clarity, we assume first that 4 divides $n$; we later explain how to adjust the definition of $\mathcal{G}_{n}$ when $n$ is not divisible by 4.) For each $2 \leq i \leq m,\left\{a_{i}, a_{i}^{\prime}, b_{i}, c_{i}\right\}$ is an independent set. The edges with both endpoints in $\left\{a_{1}, a_{1}^{\prime}, b_{1}, c_{1}\right\}$ are

$$
E_{1,1}:=\left\{\left\{a_{1}, b_{1}\right\},\left\{a_{1}, c_{1}\right\},\left\{a_{1}^{\prime}, b_{1}\right\},\left\{a_{1}^{\prime}, c_{1}\right\},\left\{b_{1}, c_{1}\right\}\right\} .
$$

The edges between the independent sets $\left\{a_{i}, a_{i}^{\prime}, b_{i}, c_{i}\right\}$ and $\left\{a_{i+1}, a_{i+1}^{\prime}, b_{i+1}, c_{i+1}\right\}$ for $1 \leq i<m$ are

$$
\begin{aligned}
E_{i, i+1}:=\{ & \left\{a_{i}, b_{i+1}\right\},\left\{a_{i}, c_{i+1}\right\},\left\{a_{i}^{\prime}, b_{i+1}\right\},\left\{a_{i}^{\prime}, c_{i+1}\right\},\left\{b_{i}, a_{i+1}\right\}, \\
& \left.\left\{b_{i}, a_{i+1}^{\prime}\right\},\left\{b_{i}, c_{i+1}\right\},\left\{c_{i}, a_{i+1}\right\},\left\{c_{i}, a_{i+1}^{\prime}\right\},\left\{c_{i}, b_{i+1}\right\}\right\} .
\end{aligned}
$$

We then let $E_{m}=\left(\cup_{i=1}^{m-1} E_{i, i+1}\right) \cup E_{1,1}$; see Figure 2(b).

Now, let

$$
M=\left\{\left\{a_{i}, b_{i+2}\right\}: i=1,2, \ldots, m-2\right\},
$$

and let $E^{(1)}, E^{(2)}, \ldots, E^{(t)}$ be all possible subsets of $M$; hence, $t=2^{m-2}$. We define $\mathcal{G}_{n}$ as

$$
\mathcal{G}_{n}=\left\{G^{(1)}=\left(V_{m}, E_{m} \cup E^{(1)}\right), G^{(2)}=\left(V_{m}, E_{m} \cup E^{(2)}\right), \ldots, G^{(t)}=\left(V_{m}, E_{m} \cup E^{(t)}\right)\right\} .
$$

Since the maximum degree of $G_{m}$ is 6, every graph in $\mathcal{G}_{n}$ has maximum degree 7. Moreover, if we let $A_{m}=\left\{a_{i}, a_{i}^{\prime}: 1 \leq i \leq m\right\}, B_{m}=\left\{b_{i}: 1 \leq i \leq m\right\}$, and $C_{m}=\left\{c_{i}: 1 \leq i \leq m\right\}$, then it is clear from our construction that $\left(A_{m}, B_{m}, C_{m}\right)$ is a tripartition for every graph in $\mathcal{G}_{n}$. An immediate consequence of this is that every graph in $\mathcal{G}_{n}$ has an $F$-coloring that assigns, for example, color 2 to every vertex in $A_{m}$, color 3 to every vertex in $B_{m}$, and a color from the independent set $I_{32}$ to every vertex in $C_{m}$. Therefore, all graphs in the family $\mathcal{G}_{n}$ are $F$-colorable.

The next theorem shows that structure learning is hard for $F$ and $\mathcal{G}_{n}$. 
TheOREM 2.6. Let $m \in \mathbb{N}^{+}$such that $m \geq 2$, and let $n=4 m$. Then, any structure learning algorithm for the constraint graph $F$ and the family of graphs $\mathcal{G}_{n}$ that succeeds with probability at least $2^{-(m-3)}$ requires at least $2^{m+1}$ samples.

Before proving this theorem, we state two key facts that will be used in its proof. In particular, Fact 2.7 shows that actually $\left(A_{m}, B_{m}, C_{m}\right)$ is the unique tripartition of $G_{m}$, and Lemma 2.8 gives a lower bound for the number of samples required by a structure learning algorithm to guarantee a prescribed success probability.

FACT 2.7. For any $m \in \mathbb{N}^{+}$, the graph $G_{m}$ is tripartite. Moreover, it has a unique tripartition $\left(A_{m}, B_{m}, C_{m}\right)$, where $A_{m}=\left\{a_{i}, a_{i}^{\prime}: 1 \leq i \leq m\right\}, B_{m}=\left\{b_{i}: 1 \leq i \leq m\right\}$, and $C_{m}=\left\{c_{i}: 1 \leq i \leq m\right\}$.

LEMMA 2.8. Let $H$ be an arbitrary constraint graph. Suppose $\hat{\mathcal{G}}=\left\{\hat{G}_{1}, \hat{G}_{2}, \ldots, \hat{G}_{r}\right\}$ is a family of $r$ distinct $H$-colorable graphs such that $H$ is identifiable with respect to $\hat{\mathcal{G}}$. Assume also that $\hat{G}_{1}$ is a subgraph of $\hat{G}_{i}$ for all $2 \leq i \leq r$ and that $\hat{G}_{r}$ is a supergraph of $\hat{G}_{i}$ for all $1 \leq i \leq r-1$. Let

$$
\eta=1-\frac{\left|\Omega_{\hat{G}_{r}}\right|}{\left|\Omega_{\hat{G}_{1}}\right|} .
$$

If there exists a structure learning algorithm for $H$ and $\hat{\mathcal{G}}$ such that for any $G^{*} \in \hat{\mathcal{G}}$, given $L$ independent samples from $\pi_{G^{*}}^{H}$ as input, it outputs $G^{*}$ with probability at least $1 / r+\alpha$ with $\alpha>0$, then $L \geq \alpha / \eta$.

We observe that when $\alpha=0$, the structure learning algorithm that outputs a graph from $\hat{\mathcal{G}}$ uniformly at random has success probability $1 / r$ without requiring any samples. We are now ready to prove Theorem 2.6.

Proof of Theorem 2.6. Let $m \geq 2$ and $n=4 m$, and for ease of notation, let $\mathcal{G}=\mathcal{G}_{n}$. Let $G^{(1)}=$ $G_{m}$ and $G^{(t)}=G_{m} \cup M$, where $M$ is defined in (1) and $t=2^{m-2}$. Hence, the graph $G^{(1)}$ (respectively, $G^{(t)}$ ) is a subgraph (respectively, supergraph) of every other graph in $\mathcal{G}$. Moreover, all graphs in $\mathcal{G}$ are distinct and $F$ is identifiable by Lemma 2.5. Hence, to apply Lemma 2.8, all we need is an upper bound for $\eta=1-\left|\Omega_{G^{(t)}}\right| /\left|\Omega_{G^{(1)}}\right|$.

By Fact 2.7, $G_{m}$ has a unique tripartition $\left(A_{m}, B_{m}, C_{m}\right)$. Since $\left\{\{1,3\},\{2,4\}, I_{32}\right\}$ is a tripartition for $F$, every $F$-coloring of $G_{m}$ induces the same tripartition of $G_{m}$. In other words, in every $F$-coloring of $G_{m}$, one of the sets $A_{m}, B_{m}$, or $C_{m}$ is colored with colors $\{1,3\}$, another is colored with $\{2,4\}$, and the third one is colored using colors from the independent set $I_{32}$ of $F$. Then, the number of $F$-colorings of $G_{m}$ such that $A_{m}$ is colored with colors from $I_{32}$ is $K \cdot 32^{2 m}$, where $K$ is the number of $F$-colorings of $B_{m} \cup C_{m}$ given a fixed $F$-coloring of $A_{m}$ that only uses colors from $I_{32}$. Observe that $K$ is the same for every $F$-coloring of $A_{m}$, and $K \geq 2$ since we can always color $B_{m}$ with color 2 and $C_{m}$ with 3, or color $B_{m}$ with 3 and $C_{m}$ with 2 . However, the number of $F$-colorings where $B_{m}$ receives colors from $I_{32}$ is at most $2 \cdot 32^{m} \cdot 2^{m} \cdot 2^{2 m}=2 \cdot 32^{m} \cdot 8^{m}$, and similarly for $C_{m}$. Hence, the probability that in a uniformly random $F$-coloring of $G_{m}, A_{m}$ is colored with colors from the independent set $I_{32}$ is at least

$$
\frac{K \cdot 32^{2 m}}{K \cdot 32^{2 m}+4 \cdot 32^{m} \cdot 8^{m}}=1-\frac{4 \cdot 8^{m}}{K \cdot 32^{m}+4 \cdot 8^{m}} \geq 1-\frac{1}{2^{2 m-1}} .
$$

Let $\sigma$ be an $F$-coloring of $G_{m}$ such that $A_{m}$ is colored with colors from $I_{32}$. Since colors from $I_{32}$ are compatible with any other color, the pair of colors $\sigma\left(a_{i}\right), \sigma\left(b_{i+2}\right)$ are compatible for any $1 \leq i \leq m-2$. Therefore, $\sigma$ is also a valid $F$-coloring of $G^{(t)}$, and thus $\sigma \in \Omega_{G^{(t)}}$. We then deduce that

$$
1-\eta=\frac{\left|\Omega_{G^{(t)}}\right|}{\left|\Omega_{G^{(1)}}\right|} \geq 1-\frac{1}{2^{2 m-1}} .
$$


Since $|\mathcal{G}|=2^{m-2}$, it follows from Lemma 2.8 that the number of samples required to learn a graph in $\mathcal{G}$ with success probability $2^{-(m-3)}$ is at least

$$
L \geq \frac{2^{-(m-3)}-2^{-(m-2)}}{\eta} \geq 2^{m+1} .
$$

Next we provide the proof of Fact 2.7; Lemma 2.8 is a direct corollary of Lemma 3.7, which is proved later.

Proof of Fact 2.7. We prove this by induction. $G_{1}$ has exactly one tripartition $\left(\left\{a_{1}, a_{1}^{\prime}\right\}, b_{1}, c_{1}\right)$. Suppose inductively that $\left(A_{m-1}, B_{m-1}, C_{m-1}\right)$ is the only tripartition of $G_{m-1}$. Since $\left\{a_{i}, b_{i-1}\right\}$, $\left\{a_{i}, c_{i-1}\right\} \in E_{m}, a_{m}$ belongs to $A_{m}$ in any tripartition of $G_{m}$. Similar statements hold for $a_{m}^{\prime}, b_{m}$, and $c_{m}$ as well. Therefore, $\left(A_{m}, B_{m}, C_{m}\right)$ is the unique tripartition of $G_{m}$.

\subsection{Proof of Theorem 1.3}

To conclude this section, we provide the proof of Theorem 1.3 from Section 1, which follows straightforwardly from Theorem 2.6.

Proof of Theorem 1.3. Let $n \geq 8$. If 4 divides $n$, then Theorem 2.6 implies that there exists a constant $c>0$ such that any structure learning algorithm for the constraint graph $F$ and the graph family $\mathcal{G}_{n}$ with success probability at least $\exp (-c n)$ ) requires at least $\exp (c n)$ samples. Since $\mathcal{G}_{n} \subseteq \mathcal{G}(n, 7)$, the result follows.

The same ideas carry over without significant modification to the case when 4 does not divide $n$. For example, suppose that $n=4 m+1$ for some $m \geq 2$. For $G \in \mathcal{G}_{4(m+1)}$, let $\hat{G}$ be the subgraph of $G$ induced by $V_{m+1} \backslash\left\{a_{m+1}^{\prime}, b_{m+1}, c_{m+1}\right\}$. We define $\mathcal{G}_{n}$ as the family of the subgraphs $\hat{G}$ for each $G \in \mathcal{G}_{4(m+1)}$. When $n=4 m+2$ or $n=4 m+3$, we consider instead the graph families of the subgraphs induced by $V_{m+1} \backslash\left\{b_{m+1}, c_{m+1}\right\}$ and $V_{m+1} \backslash\left\{c_{m+1}\right\}$, respectively. The argument in the proof of Theorem 2.6 carries over to these graph families straightforwardly. Since in every case $\mathcal{G}_{n} \subseteq \mathcal{G}(n, 7)$, the result follows.

\section{LEARNING PROPER $q$-COLORINGS}

In this section, we consider statistical identifiability, structure learning, and equivalent-structure learning for proper $q$-colorings, where $H=K_{q}$ and $\pi_{G}$ is the uniform distribution over the proper $q$-colorings of the graph $G$. In particular, we prove Theorem 1.4 from Section 1.

\subsection{A Structure Learning Algorithm}

In this section, we introduce a general structure learning algorithm for any constraint graph $H$ with at least one hard constraint (i.e., $H \neq K_{q}^{+}$). In Section 3.2, we analyze its running time and sample complexity for proper colorings. Later in Sections 4 and 6.3, we consider more general settings where this algorithm is also efficient.

Fix $H \neq K_{q}^{+}$and suppose $\{i, j\} \notin E(H)$. Given independent samples $\sigma^{(1)}, \ldots, \sigma^{(L)}$ from $\pi_{G}=\pi_{G}^{H}$ for some unknown graph $G=(V, E)$, the algorithm checks for every pair of vertices $u, v \in V$ whether there is at least one sample $\sigma^{(k)}$ such that $\sigma_{u}^{(k)}=i$ and $\sigma_{v}^{(k)}=j$. If this is the case, then the edge $\{u, v\}$ does not belong to $E$. Otherwise, the algorithm adds the edge $\{u, v\}$ to the estimator $\hat{E}$ of $E$. This structure learning algorithm, which we call STRUCTLEARN-H, has running time $O\left(L n^{2}\right)$ and was used before in Bresler et al. [6] for the hard-core model. The effectiveness of STRUCTLEARN-H depends crucially on how likely are non-adjacent vertices to receive colors $i$ and $j$. For $v \in V$, let $X_{v}$ be the random variable for the color of $v$ under $\pi_{G}$. 
Lemma 3.1. Let $H \neq K_{q}^{+}$and $\{i, j\} \notin E(H)$. Suppose that for all $\{u, v\} \notin E$,

$$
\operatorname{Pr}\left[X_{u}=i, X_{v}=j\right] \geq \delta
$$

for some $\delta>0$. Let $\hat{G}=(V, \hat{E})$ be the output of the algorithm STRUCTLEARN-H. Then, for all $\varepsilon \in(0,1)$, $\operatorname{Pr}[E=\hat{E}] \geq 1-\varepsilon$ provided $L \geq \delta^{-1} \log \left(\frac{n^{2}}{2 \varepsilon}\right)$.

Proof. Suppose $\{u, v\} \notin E$, and let $Z_{u v}$ be the number of samples where vertices $u$ and $v$ are assigned colors $i$ and $j$, respectively. Since $\operatorname{Pr}\left[X_{u}=i, X_{v}=j\right] \geq \delta$, we get

$$
\operatorname{Pr}\left[Z_{u v}=0\right] \leq(1-\delta)^{L} \leq \mathrm{e}^{-\delta L} \leq \frac{2 \varepsilon}{n^{2}} .
$$

The result follows from a union bound over the pairs of non-adjacent vertices.

\subsection{Efficient Structure Learning When $q \geq d+1$}

In this section, we prove part (1) of Theorem 1.4. We show that for proper $q$-colorings with $q \geq$ $d+1$ and any graph in $\mathcal{G}(n, d)$, the structure learning algorithm STRUCTLEARN-H (see Section 3.1) requires $O\left(q d^{3} \log (n / \varepsilon)\right)$ samples to succeed with probability at least $1-\varepsilon$ and has running time $O\left(q d^{3} n^{2} \log (n / \varepsilon)\right)$. This can be deduced immediately from the next lemma and Lemma 3.1, since $H=K_{q} \neq K_{q}^{+}$in this setting.

Lemma 3.2. Suppose that $q \geq d+1$, and let $\{u, v\} \notin E$. Then

$$
\operatorname{Pr}\left[X_{u}=X_{v}\right] \geq \frac{1}{q(d+1)^{3}} .
$$

Proof. Let $u, v \in V$ be such that $\{u, v\} \notin E$, and let $\partial u, \partial v$ denote the neighborhoods of $u$ and $v$, respectively, which may overlap. Let $G^{\prime}$ be $G$ with the vertices $u, v$ removed (the edges adjacent to $u$ and $v$ are removed as well).

Let $A(c, s, t)$ be the set of colorings of $G^{\prime}$ where color $c$ appears exactly $s$ times in $\partial u$ and exactly $t$ times in $\partial v$. Given a coloring in $A(c, s, t)$, we produce a coloring in $A(c, 0,0)$ as follows. List the positions where $c$ occurs in $\partial u \cup \partial v$ and then re-color the vertices in the order of the list. Note that every vertex $w$ in $\partial u \cup \partial v$ has at least two colors that do not occur in its neighborhood since in $G^{\prime}$ the vertex $w$ has degree at most $d-1$ (recall that we removed $u$ and $v$ from $G$ to obtain $G^{\prime}$ ). This maps at most $d^{s+t}$ colorings from $A(c, s, t)$ to a coloring in $A(c, 0,0)$ (given the list of positions, we can recover the original coloring; there are at most $d^{s+t}$ lists where we first list the vertices in $\partial u$ and then vertices in $\partial v \backslash \partial u$ ). Hence, we have

$$
|A(c, 0,0)| \geq \frac{|A(c, s, t)|}{d^{s+t}} .
$$

Let $A(c, \leq j, \leq k):=\sum_{s \leq j, t \leq k} A(c, s, t)$, and let $\mu$ be the uniform distribution over the colorings of $G^{\prime}$. We claim that in any coloring there exist at least $q-d$ colors that satisfy the following: $c$ occurs at most once in $\partial u$ and at most once in $\partial v$. Indeed, adding over all colors the number of occurrences in $\partial u$ and the number of occurrences in $\partial v$, we can get at most $2 d$; thus, at most $d$ colors can occur at least twice in $\partial u$ or at least twice in $\partial v$. Thus,

$$
\mu(A(1, \leq 1, \leq 1))+\mu(A(2, \leq 1, \leq 1))+\cdots+\mu(A(q, \leq 1, \leq 1)) \geq q-d,
$$

and by symmetry, $\mu(A(1, \leq 1, \leq 1)) \geq \frac{q-d}{q} \geq \frac{1}{d+1}$. Since

$$
\mu(A(1, \leq 1, \leq 1))=\sum_{i, j \in\{0,1\}} \mu(A(1, i, j)),
$$


from (2) we get

$$
\mu(A(1,0,0)) \geq \frac{1}{(d+1)^{3}} .
$$

This means that with probability at least $1 /(d+1)^{3}$, color 1 does not occur in $\partial u \cup \partial v$ in a random coloring of $G^{\prime}$.

Consider generating a uniformly random coloring of $G$ using rejection sampling as follows. Pick a uniformly random coloring of $G^{\prime}$, a uniformly random color $c_{1}$ for $u$, and a uniformly random color $c_{2}$ for $v$. If the resulting coloring is valid for $G$, then accept and otherwise reject. Since in every round each coloring has the same probability of being picked, the generated coloring is a uniformly random coloring of $G$. Notice that with probability $1 / q^{2}$, we propose $c_{1}=c_{2}=1$ in the rejection sampling procedure, and hence with probability at least $\frac{1}{q^{2}(d+1)^{3}}$, our process accepts and produces a coloring of $G$ where $u, v$ both receive color 1 . Thus, in a uniformly random coloring of $G$ vertices, $u, v$ receive color 1 with probability at least $\frac{1}{q^{2}(d+1)^{3}}$, and by symmetry, the probability that they receive the same color is at least $\frac{1}{q(d+1)^{3}}$, as claimed.

\subsection{Identifiability for Proper $q$-Colorings}

In this section, we prove part (2) of Theorem 1.4. We show that when $q \leq d$ there exist two distinct graphs $G, G^{\prime} \in \mathcal{G}(n, d)$ such that $\pi_{G}=\pi_{G^{\prime}}$, or equivalently that $G$ and $G^{\prime}$ have the same set of $q$-colorings.

Theorem 3.3. Let $n, q, d \in \mathbb{N}^{+}$such that $q \leq d$ and $n \geq q+2$. Then, the structure learning problem for $q$-colorings is not identifiable with respect to the family of graphs $\mathcal{G}(n, d)$.

Proof. Let $G=(V, E)$ be a graph with

$$
V=\left\{c_{1}, \ldots, c_{q-1}, u, v, w_{1}, \ldots, w_{n-q-1}\right\},
$$

where $\left\{c_{1}, \ldots, c_{q-1}, u, v\right\}$ is a clique of size $q+1$ except for the one edge $\{u, v\}$ that is not in $E$, and $\left\{w_{1}, \ldots, w_{n-q-1}\right\}$ is a simple path from $w_{1}$ to $w_{n-q-1}$. $G$ has one additional edge connecting $v$ and $w_{1}$. Then, in every $q$-coloring of $G$, the vertices $u$ and $v$ receive the same color, and thus $u$ and $w_{1}$ are assigned distinct colors. Hence, the graph $G$ and the graph $G^{\prime}=\left(V, E \cup\left\{u, w_{1}\right\}\right)$ have the same set of $q$-colorings. Since both $G$ and $G^{\prime}$ are $n$-vertex graphs of maximum degree at most $q \leq d$, the structure learning for $q$-colorings is not identifiable with respect to $\mathcal{G}(n, d)$.

\subsection{Strong Lower Bound When $q \leq d-\sqrt{d}+\Theta(1)$}

In this section, we prove part (3) of Theorem 1.4, establishing a strong learning lower bound for proper colorings when $q \leq d-\sqrt{d}+\Theta(1)$.

As defined previously, an equivalent-structure learning algorithm for a graph family $\mathcal{G}$ finds a graph $\hat{G} \in \mathcal{G}$ such that $\Omega_{G}=\Omega_{\hat{G}}$, where $G \in \mathcal{G}$ is the actual hidden graph. We exhibit a family of graphs of maximum degree $q+[\sqrt{q}\rceil-1$ such that every graph in the family has almost the same set of $q$-colorings. This makes equivalent-structure learning hard in this family. We use this fact to prove part (3) of Theorem 1.4.

First, we define a graph $\Gamma_{m, t}=\left(V\left(\Gamma_{m, t}\right), E\left(\Gamma_{m, t}\right)\right)$, which is the base of all graphs in our graph family. For any $m, t \in \mathbb{N}^{+}$with $t<q$, the graph $\Gamma_{m, t}$ is defined as follows. Let $C_{1}, \ldots, C_{m}$ be cliques of size $q-1$, and let $I_{1}, \ldots, I_{m}$ be independent sets of size $t$. Then,

$$
V\left(\Gamma_{m, t}\right)=\bigcup_{i=1}^{m}\left(V\left(C_{i}\right) \cup V\left(I_{i}\right)\right),
$$




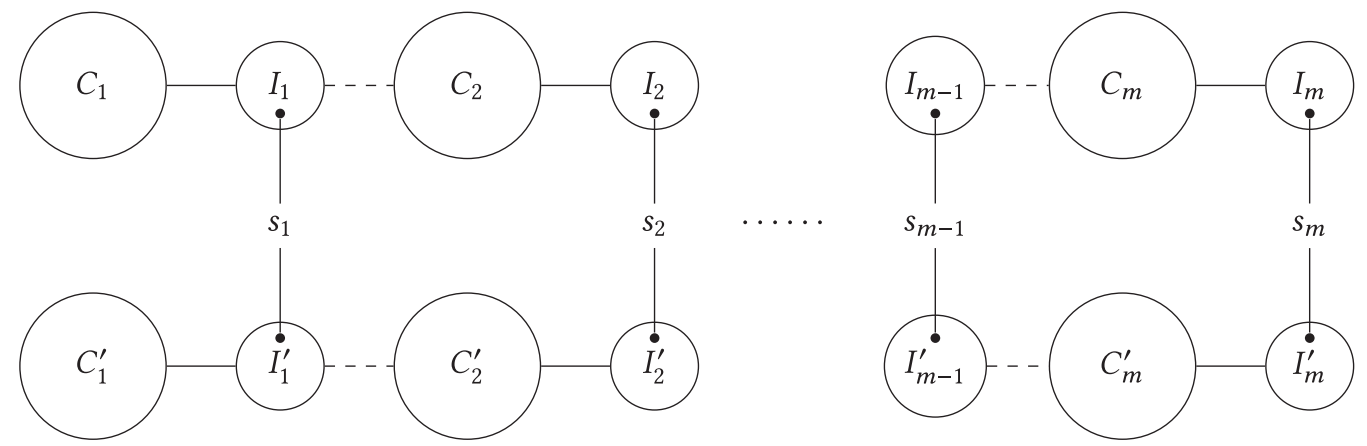

Fig. 3. The graph $G_{m, t}$. Each of $C_{1}, \ldots, C_{m}, C_{1}^{\prime}, \ldots, C_{m}^{\prime}$ is a clique of size $q-1$, and each of $I_{1}, \ldots, I_{m}, I_{1}^{\prime}, \ldots, I_{m}^{\prime}$ is an independent set of size $t<q$. Solid lines between $C_{i}\left(C_{i}^{\prime}\right)$ and $I_{i}\left(I_{i}^{\prime}\right)$ mean that every vertex in $C_{i}\left(C_{i}^{\prime}\right)$ is adjacent to every vertex in $I_{i}\left(I_{i}^{\prime}\right)$. Dashed lines between $I_{i-1}\left(I_{i-1}^{\prime}\right)$ and $C_{i}\left(C_{i}^{\prime}\right)$ mean that every vertex in $I_{i-1}\left(I_{i-1}^{\prime}\right)$ is adjacent to roughly $(q-1) / t$ vertices in $C_{i}\left(C_{i}^{\prime}\right)$ with no two vertices in $I_{i-1}$ $\left(I_{i-1}^{\prime}\right)$ sharing a common neighbor in $C_{i}\left(C_{i}^{\prime}\right)$. Each vertex $s_{i}$ is adjacent to exactly one vertex from $I_{i}$ and one from $I_{i}^{\prime}$.

where $V\left(C_{i}\right), V\left(I_{i}\right)$ are the vertices of $C_{i}, I_{i}$, respectively. In addition to the edges in the cliques $C_{i}$ for $1 \leq i \leq m, E\left(\Gamma_{m, t}\right)$ contains the following edges:

(1) For $1 \leq i \leq m$, there is a complete bipartite graph between $C_{i}$ and $I_{i}$. In other words, for $v \in C_{i}$ and $u \in I_{i},\{v, u\} \in E\left(\Gamma_{m, t}\right)$.

(2) For $2 \leq i \leq m$, each $C_{i}$ is partitioned into $t$ almost equally sized disjoint subsets $C_{i, 1}, \ldots, C_{i, t}$ of size either $\lfloor(q-1) / t\rfloor$ or $\lceil(q-1) / t\rceil$. Then, the $j$-th vertex of $I_{i-1}$ is connected to every vertex in $C_{i, j}$.

For $1 \leq i \leq m-1$, pick $u_{i}$ to be a vertex from $I_{i}$ that is adjacent to exactly $\lfloor(q-1) / t\rfloor$ vertices in $C_{i}$ and pick $u_{m}$ arbitrarily from $I_{m}$. We refer to these vertices $u_{1}, \ldots, u_{m}$ as ports of the graph $\Gamma_{m, t}$. The key fact about the graph $\Gamma_{m, t}$ that allows us to construct a graph family with the desired properties is the following.

FACT 3.4. Let $q, m, t \in \mathbb{N}^{+}$such that $q \geq 3$ and $t<q$. In every $q$-coloring of $\Gamma_{m, t}$, all cliques $C_{1}, \ldots, C_{m}$ are colored by the same set of $q-1$ colors, and all independent sets $I_{1}, \ldots, I_{m}$ are colored with the remaining color.

Fact 3.4, which is proved later, implies that all $q$-colorings of the graph $\Gamma_{m, t}$ can be classified by the color of $I_{1}$.

Next we define a graph $G_{m, t}=\left(V_{m, t}, E_{m, t}\right)$, and every graph in our graph family will be a subgraph of $G_{m, t}$. Take the graph $\Gamma_{m, t}=\left(V\left(\Gamma_{m, t}\right), E\left(\Gamma_{m, t}\right)\right)$ and a copy of it $\Gamma_{m, t}^{\prime}=\left(V\left(\Gamma_{m, t}^{\prime}\right), E\left(\Gamma_{m, t}^{\prime}\right)\right)$. The ports of $\Gamma_{m, t}$ and $\Gamma_{m, t}^{\prime}$ are denoted by $u_{1}, \ldots, u_{m}$ and $u_{1}^{\prime}, \ldots, u_{m}^{\prime}$, respectively. Moreover, let $s_{1}, \ldots, s_{m}$ be $m$ additional vertices. Then,

$$
V_{m, t}=V\left(\Gamma_{m, t}\right) \cup V\left(\Gamma_{m, t}^{\prime}\right) \cup\left\{s_{1}, \ldots, s_{m}\right\}
$$

and

$$
E_{m, t}=E\left(\Gamma_{m, t}\right) \cup E\left(\Gamma_{m, t}^{\prime}\right) \cup\left\{\left\{u_{i}, s_{i}\right\},\left\{u_{i}^{\prime}, s_{i}\right\}: 1 \leq i \leq m\right\} ;
$$

see Figure 3 for an illustration of the graph $G_{m, t}$.

The family of graphs $\mathcal{G}_{m, t}$ is defined as follows. All graphs in this family are distinct and are subgraphs of $G_{m, t}$. Assume $m \in \mathbb{N}^{+}$is even. Let

$$
M=\left\{\left\{u_{i}^{\prime}, s_{i}\right\}: m / 2<i \leq m\right\} .
$$


Let $E^{(1)}, \ldots, E^{(l)}$ be all of the subsets of $M$; hence, $l=2^{m / 2}$. We let

$$
\mathcal{G}_{m, t}=\left\{G^{(j)}=\left(V_{m, t}, E_{m, t} \backslash E^{(j)}\right): 1 \leq j \leq l\right\} .
$$

The graphs in $\mathcal{G}_{m, t}$ satisfy the following property, which we prove later.

FACт 3.5. If $G=(V, E) \in \mathcal{G}_{m, t}$, then $|V|=(2 q+2 t-1) m$ and the maximum degree of $G$ is at most

$$
q-1+\max \{t,\lceil q / t\rceil\} \text {. }
$$

Using Fact 3.4 and ideas similar to those in the proof of Theorem 2.6, we can show that both structure and equivalent-structure learning are computationally hard in $\mathcal{G}_{m, t}$ (sample complexity is exponential in $m$ ). This immediately implies that structure learning is also hard in $\mathcal{G}(n, d)$ provided $d$ is large enough so that $\mathcal{G}_{m, t} \subseteq \mathcal{G}(n, d)$. However, this does not necessarily imply that equivalent-structure learning is hard for $\mathcal{G}(n, d)$, which is our goal. For this, we introduce instead a larger graph family $\mathcal{F}_{m, t}$ that contains $\mathcal{G}_{m, t}$. Suppose $d$ is an integer such that

$$
d \geq q-1+\max \{t,\lceil q / t\rceil\},
$$

the maximum degree of any graph in $\mathcal{G}_{m, t}$; see Fact 3.5. The graph family $\mathcal{F}_{m, t}$ contains all graphs in $\mathcal{G}(n, d)$ that have the same set of colorings as some graph in $\mathcal{G}_{m, t}$. Namely,

$$
\mathcal{F}_{m, t}=\left\{G \in \mathcal{G}(n, d): \Omega_{G}=\Omega_{G^{\prime}}, G^{\prime} \in \mathcal{G}_{m, t}\right\},
$$

and $\mathcal{G}_{m, t} \subseteq \mathcal{F}_{m, t} \subseteq \mathcal{G}(n, d)$ by definition.

The next theorem establishes a lower bound for any equivalent-structure learning algorithm for $\mathcal{F}_{m, t}$. We will later see that this lower bound applies also to the graph family $\mathcal{G}(n, d)$, which establishes part (3) of Theorem 1.4.

Theorem 3.6. Let $q, m, t \in \mathbb{N}^{+}$such that $q \geq 3, m$ is even, and $t<q$. Any equivalent-structure learning algorithm for proper vertex $q$-colorings and the graph family $\mathcal{F}_{m, t}$ that succeeds with probability at least $q \cdot \exp [-m /(4(q-1))]$ requires at least $\exp [m /(4(q-1))]$ samples.

As we shall see, the assumption that $m$ is even is not essential but makes the calculations in the proof of Theorem 3.6 clearer. The following generalization of Lemma 2.8 will be used in the proof of Theorem 3.6.

Lemma 3.7. Let $H$ be an arbitrary constraint graph, and let $\mathcal{F}_{1}, \ldots, \mathcal{F}_{r}$ be $r$ families of distinct $H$-colorable graphs. Suppose that for all $1 \leq i \leq r$, every graph in $\mathcal{F}_{i}$ has the same set of $H$-colorings $\Omega_{\mathcal{F}_{i}}$. Assume also that $\Omega_{\mathcal{F}_{i}} \neq \Omega_{\mathcal{F}_{j}}$ for $i \neq j$ and that $\Omega_{\mathcal{F}_{r}} \subseteq \Omega_{\mathcal{F}_{i}} \subseteq \Omega_{\mathcal{F}_{1}}$ for all $1 \leq i \leq r$. Let $\hat{\mathcal{F}}=$ $\mathcal{F}_{1} \cup \cdots \cup \mathcal{F}_{r}$, and let

$$
\eta=1-\frac{\left|\Omega_{\mathcal{F}_{r}}\right|}{\left|\Omega_{\mathcal{F}_{1}}\right|} .
$$

If there exists an equivalent-structure learning algorithm for $H$ and $\hat{\mathcal{F}}$ such that for any $G^{*} \in \hat{\mathcal{F}}$, given $L$ independent samples from $\pi_{G^{*}}^{H}$ as input, it outputs a graph $G$ satisfying $\Omega_{G}=\Omega_{G^{*}}$ with probability at least $1 / r+\alpha$ with $\alpha>0$, then $L \geq \alpha / \eta$.

We remark that Lemma 2.8 corresponds to the special case where each family $\mathcal{F}_{i}$ contains a single graph, and thus it follows immediately from Lemma 3.7. We are now ready to prove Theorem 3.6.

Proof of Theorem 3.6. Let $G^{(1)}=G_{m, t} \backslash M=\left(V_{m, t}, E_{m, t} \backslash M\right)$ and $G^{(l)}=G_{m, t}$, where $M$ is as in (3) and $l=2^{m / 2}$. Let $\mathcal{F}_{j}$ be the class of graphs that contains all graphs in $\mathcal{F}_{m, t}$ that has the same set of colorings as $G^{(j)}$. (Recall that $\mathcal{F}_{m, t}$ is the set of graphs in $\mathcal{G}(n, d)$ that has the same set of 
colorings as some graph in $\mathcal{G}_{m, t}$.) Let $\Omega_{\mathcal{F}_{j}}=\Omega_{G^{(j)}}$ for all $j$. Note that $\Omega_{\mathcal{F}_{l}} \subseteq \Omega_{\mathcal{F}_{j}} \subseteq \Omega_{\mathcal{F}_{1}}$ for all $1 \leq j \leq l$. Let

$$
\eta=1-\frac{\left|\Omega_{\mathcal{F}_{l}}\right|}{\left|\Omega_{\mathcal{F}_{1}}\right|} .
$$

We establish an upper bound on $\eta$ and then apply Lemma 3.7 to prove the theorem.

By Fact 3.4, all $q$-colorings of $G^{(1)}=G_{m, t} \backslash M$ can be classified by the colors of the independent sets $I_{1}, I_{1}^{\prime}$ and of the vertices $s_{1}, \ldots, s_{m}$. Then, the number of $q$-colorings of $G^{(1)}$ where all vertices in $I_{1}$ and $I_{1}^{\prime}$ receive the same color is equal to $q(q-1)^{m}[(q-1) !]^{2 m}$, since there are $q$ choices for the color of $I_{1}$ and $I_{1}^{\prime}, q-1$ choices for the color of each $s_{i}$, and $(q-1)$ ! colorings for each $C_{i}$ and $C_{i}^{\prime}$. Similarly, the number of colorings where $I_{1}$ and $I_{1}^{\prime}$ receive distinct colors is equal to $q(q-1)(q-2)^{m / 2}(q-1)^{m / 2}[(q-1) !]^{2 m}$, since there are $q-2$ choices for the color of $s_{i}$ for $1 \leq$ $i \leq m / 2$ while $q-1$ choices for $m / 2<i \leq m$. Thus, the probability that in a uniform random $q$ coloring of $G^{(1)}$ the vertices in $I_{1}$ and $I_{1}^{\prime}$ have the same color is

$$
\begin{aligned}
\frac{q(q-1)^{m}[(q-1) !]^{2 m}}{q(q-1)^{m}[(q-1) !]^{2 m}+q(q-1)(q-2)^{m / 2}(q-1)^{m / 2}[(q-1) !]^{2 m}}=1-\frac{(q-2)^{m / 2}}{(q-1)^{m / 2-1}+(q-2)^{m / 2}} \\
\geq 1-(q-1)\left(\frac{q-2}{q-1}\right)^{m / 2} \geq 1-(q-1) \mathrm{e}^{-\frac{m}{2(q-1)}} .
\end{aligned}
$$

Let $\sigma$ be a $q$-coloring of $G^{(1)}$ where $I_{1}$ and $I_{1}^{\prime}$ receive the same color. Then, Fact 3.4 implies that $\sigma\left(u_{i}^{\prime}\right) \neq \sigma\left(s_{i}\right)$ for all $m / 2<i \leq m$, since $I_{i}$ and $s_{i}$ have distinct colors and $I_{i}$ and $I_{i}^{\prime}$ have the same color. Therefore, $\sigma$ is a proper $q$-coloring of $G^{(l)}=G_{m, t}$; hence, $\sigma \in \Omega_{\mathcal{F}_{l}}$ and

$$
\frac{\left|\Omega_{\mathcal{F}_{l}}\right|}{\left|\Omega_{\mathcal{F}_{1}}\right|}=\operatorname{Pr}_{\pi_{\mathcal{F}_{1}}}\left[\sigma \in \Omega_{\mathcal{F}_{l}}\right] \geq \operatorname{Pr}_{\pi_{\mathcal{F}_{1}}}\left[\sigma\left(I_{1}\right)=\sigma\left(I_{1}^{\prime}\right)\right] \geq 1-(q-1) \mathrm{e}^{-\frac{m}{2(q-1)}}
$$

(Note that $\pi_{\mathcal{F}_{1}}=\pi_{G^{(1)}}=\pi_{G_{m, t} \backslash M}$.) Then,

$$
\eta=1-\frac{\left|\Omega_{\mathcal{F}_{l}}\right|}{\left|\Omega_{\mathcal{F}_{1}}\right|} \leq(q-1) \mathrm{e}^{-\frac{m}{2(q-1)}}
$$

Every graph in $\mathcal{G}_{m, t}$ is a distinct subgraph of $G_{m, t}$ and $\left|\mathcal{G}_{m, t}\right|=2^{m / 2}$. Moreover, for any $G=$ $(V, E) \in \mathcal{G}_{m, t}$ and any $m / 2<i \leq m$ such that $\left\{u_{i}^{\prime}, s_{i}\right\} \notin E$, there are $q$-colorings of $G$ where $u_{i}^{\prime}$ and $s_{i}$ are assigned the same color. Consequently, for any $G, G^{\prime} \in \mathcal{G}_{m, t}$, we have $\Omega_{G} \neq \Omega_{G^{\prime}}$ whenever $G \neq G^{\prime}$. Then, $\Omega_{\mathcal{F}_{i}} \neq \Omega_{\mathcal{F}_{j}}$ for any $i \neq j$, and by definition, all graphs in $\mathcal{F}_{i}$ are distinct for each $i$. Therefore, Lemma 3.7 implies that to equivalently learn any $G \in \mathcal{F}_{m, t}$ with probability at least $2^{-m / 2}+\alpha$, the number of random samples needed is $L \geq \alpha / \eta$. Setting

$$
\alpha=q \mathrm{e}^{-\frac{m}{4(q-1)}}-2^{-m / 2}>0
$$

we get that to equivalently learn a graph $G \in \mathcal{F}_{m, t}$ with success probability at least $q \mathrm{e}^{-\frac{m}{4(q-1)}}$, we require

$$
L \geq \frac{q \mathrm{e}^{-\frac{m}{4(q-1)}}-2^{-m / 2}}{(q-1) \mathrm{e}^{-\frac{m}{2(q-1)}}} \geq \frac{q \mathrm{e}^{-\frac{m}{4(q-1)}}-\mathrm{e}^{-\frac{m}{4(q-1)}}}{(q-1) \mathrm{e}^{-\frac{m}{2(q-1)}}} \geq \mathrm{e}^{\frac{m}{4(q-1)}}
$$

where the second inequality follows from $2 \geq \mathrm{e}^{\frac{1}{2(q-1)}}$ when $q \geq 3$.

The following corollary of Theorem 3.6 corresponds to part (3) of Theorem 1.4.

Corollary 3.8. Let $q, n, d \in \mathbb{N}^{+}$such that $q \geq 3, d \geq q+\lceil\sqrt{q}\rceil-1$ and $n \geq 4 q+4\lceil\sqrt{q}\rceil-2$. Then, there exists a constant $c>0$ such that any equivalent-structure learning algorithm for $q$ colorings and the graph family $\mathcal{G}(n, d)$ that succeeds with probability at least $\exp (-c n)$ requires at least $\exp (\mathrm{cn})$ samples. 
Proof. Let $t=\lceil\sqrt{q}\rceil$ and $k=2 q+2 t-1$. If $2 k$ divides $n$, then take $m=n / k$ so that $m$ is even. By Fact 3.5, every graph in $\mathcal{G}_{m, t}$ has $n=m k$ vertices and maximum degree

$$
q-1+\max \{t,\lceil q / t\rceil\} \leq q-1+\max \{[\sqrt{q}\rceil,[q / \sqrt{q}\rceil\}=q+\lceil\sqrt{q}\rceil-1,
$$

and thus $\mathcal{G}_{m, t} \subseteq \mathcal{F}_{m, t} \subseteq \mathcal{G}(n, d)$ provided $d \geq q+\lceil\sqrt{q}\rceil-1$. Theorem 3.6 implies that there exists $c=c(q)>0$ such that any equivalent-structure learning algorithm for $\mathcal{F}_{m, t}$ with success probability at least $\exp (-c n)$ requires $\exp (c n)$ samples. By definition, the set of $q$-colorings of any graph in $\mathcal{G}(n, d) \backslash \mathcal{F}_{m, t}$ is distinct from the set of $q$-colorings of any graph in $\mathcal{F}_{m, t}$. Since also $\mathcal{F}_{m, t} \subseteq \mathcal{G}(n, d)$, equivalent-structure learning in $\mathcal{G}(n, d)$ is strictly harder than in $\mathcal{F}_{m, t}$. Especially, any equivalentstructure learning algorithm for $\mathcal{G}(n, d)$ with success probability at least $\exp (-c n)$ requires at least $\exp (c n)$ samples. Note that $d \geq q+\lceil\sqrt{q}\rceil-1$ is equivalent to $q \leq d-\sqrt{d}+\Theta(1)$.

The result follows in similar fashion when $2 k$ does not divide $n$, but we are required to modify slightly the graph families $\mathcal{G}_{m, t}$ and $\mathcal{F}_{m, t}$. Suppose $n=k m+r$, where $m$ is even and $1 \leq r<2 k$, and let $W=\left\{w_{1}, \ldots, w_{r}\right\}$ be a simple path. For every $G \in \mathcal{G}_{m, t}$, add $W$ and the edge $\left\{s_{m}, w_{1}\right\}$ to $G$ to obtain a graph $\hat{G}$. Let $\hat{\mathcal{G}}_{m, t}$ be the resulting graph family. Every graph in $\hat{\mathcal{G}}_{m, t}$ has exactly $n$ vertices and maximum degree $q+\lceil\sqrt{q}\rceil-1$. Moreover, every $q$-coloring of $G \in \mathcal{G}_{m, t}$ corresponds to exactly $(q-1)^{r}$ colorings of $\hat{G} \in \hat{\mathcal{G}}_{m, t}$. Define $\hat{\mathcal{F}}_{m, t}$ as before-that is, $\hat{\mathcal{F}}_{m, t}$ is the set of all graphs in $\mathcal{G}(n, d)$ that have the same set of colorings as some graph in $\hat{\mathcal{G}}_{m, t}$. The argument in the proof of Theorem 3.6 and Lemma 3.7 implies that any equivalent-structure learning algorithm for $\hat{\mathcal{F}}_{m, t}$ with success probability at least $\exp (-c n)$ requires $\exp (c n)$ independent samples, where $c=c(q)>0$ is a suitable constant. Since $\hat{\mathcal{G}}_{m, t} \subseteq \hat{\mathcal{F}}_{m, t} \subseteq \mathcal{G}(n, d)$ for $d \geq q+\lceil\sqrt{q}\rceil-1$, the result follows.

We conclude this section with the proofs of Fact 3.4, Fact 3.5, and Lemma 3.7.

Proof OF FACT 3.4. Let $\sigma$ be a $q$-coloring of $\Gamma_{m, t}$. For $1 \leq i<m$, since every vertex in $I_{i}$ is adjacent to every vertex in $C_{i}$, all vertices in $I_{i}$ have the same color in $\sigma$, which is the one color not used by $C_{i}$. Moreover, every vertex in $C_{i+1}$ is adjacent to a vertex of $I_{i}$, and thus $C_{i+1}$ is colored with the same set of $q-1$ colors as $C_{i}$ in $\sigma$. Then, every clique $C_{1}, \ldots, C_{m}$ is colored with the same set of $q-1$ colors, and every independent set $I_{1}, \ldots, I_{m}$ is colored with the one remaining color in $\sigma$.

Proof of FaCt 3.5. The number of vertices in $G_{m, t}$ is $m\left(\left|C_{1}\right|+\left|C_{1}^{\prime}\right|+\left|I_{1}\right|+\left|I_{1}^{\prime}\right|+1\right)=(2 q+2 t-$ 1) $m$. The degree of the vertices in the cliques $C_{i}$ or $C_{i}^{\prime}$ is at most $q-2+t+1=q-1+t$. The degree of non-port vertices in the independent sets $I_{i} \backslash\left\{u_{i}\right\}$ or $I_{i}^{\prime} \backslash\left\{u_{i}^{\prime}\right\}$ is at most $q-1+\lceil(q-1) / t\rceil$. The degree of $u_{i}$ or $u_{i}^{\prime}$ is at most $q-1+\lfloor(q-1) / t\rfloor+1$. Thus, the maximum degree of $G_{m, t}$ is no more than

$$
q-1+\max \{t,\lceil(q-1) / t\rceil,\lfloor(q-1) / t\rfloor+1\} \leq q-1+\max \{t,[q / t\rceil\},
$$

where we use $\lceil a / b\rceil \leq\lfloor a / b\rfloor+1=\lceil(a+1) / b\rceil$ for any integer $a, b \in \mathbb{N}^{+}$. Since every graph in the family $\mathcal{G}_{m, t}$ is a subgraph of $G_{m, t}$, the same upper bound on maximum degree holds.

Proof of Lemma 3.7. Let $\mathcal{A}$ be any (possibly randomized) equivalent-structure learning algorithm that, given $L$ independent samples $\Gamma=\left(\sigma^{(1)}, \ldots, \sigma^{(L)}\right) \in\left(\Omega_{\mathcal{F}_{i}}\right)^{L}$ from an unknown distribution $\pi_{\mathcal{F}_{i}}=\pi_{G^{*}}$ for some $G^{*} \in \mathcal{F}_{i}$, outputs a graph $\mathcal{A}(\Gamma)$ in $\hat{\mathcal{F}}$. For any $G^{*}$, the probability that $\mathcal{A}$ equivalently learns the graph given $L$ independent samples from $\pi_{\mathcal{F}_{i}}$ is

$$
\operatorname{Pr}\left[\mathcal{A}(\Gamma) \in \mathcal{F}_{i}\right]=\sum_{x \in\left(\Omega_{\mathcal{F}_{i}}\right)^{L}} \operatorname{Pr}[\Gamma=x] \operatorname{Pr}\left[\mathcal{A}(x) \in \mathcal{F}_{i}\right]
$$


Recall that by assumption, $\Omega_{\mathcal{F}_{r}} \subseteq \Omega_{\mathcal{F}_{i}}$. Let $\mathcal{T}$ be the set of all sample sequences $\sigma^{(1)}, \ldots, \sigma^{(L)}$ such that $\sigma^{(j)} \notin \Omega_{\mathcal{F}_{r}}$ for at least one $j$; namely, $\mathcal{T}=\left(\Omega_{\mathcal{F}_{i}}\right)^{L} \backslash\left(\Omega_{\mathcal{F}_{r}}\right)^{L}$. Note that

$$
|\mathcal{T}|=\left|\Omega_{\mathcal{F}_{i}}\right|^{L}-\left|\Omega_{\mathcal{F}_{r}}\right|^{L} .
$$

Then,

$$
\begin{aligned}
\operatorname{Pr}\left[A(\Gamma) \in \mathcal{F}_{i}\right] & =\sum_{x \in\left(\Omega_{\mathcal{F}_{r}}\right)^{L}} \frac{1}{\left|\Omega_{\mathcal{F}_{i}}\right|^{L}} \cdot \operatorname{Pr}\left[\mathcal{A}(x) \in \mathcal{F}_{i}\right]+\sum_{x \in \mathcal{T}} \frac{1}{\left|\Omega_{\mathcal{F}_{i}}\right|^{L}} \cdot \operatorname{Pr}\left[\mathcal{A}(x) \in \mathcal{F}_{i}\right] \\
& \leq \frac{1}{\left|\Omega_{\mathcal{F}_{r}}\right|^{L}} \sum_{x \in\left(\Omega_{\mathcal{F}_{r}}\right)^{L}} \operatorname{Pr}\left[\mathcal{A}(x) \in \mathcal{F}_{i}\right]+\frac{\left|\Omega_{\mathcal{F}_{i}}\right|^{L}-\left|\Omega_{\mathcal{F}_{r}}\right|^{L}}{\left|\Omega_{\mathcal{F}_{i}}\right|^{L}} .
\end{aligned}
$$

Since $\Omega_{\mathcal{F}_{i}} \subseteq \Omega_{\mathcal{F}_{1}}$, we get

$$
\frac{\left|\Omega_{\mathcal{F}_{i}}\right|^{L}-\left|\Omega_{\mathcal{F}_{r}}\right|^{L}}{\left|\Omega_{\mathcal{F}_{i}}\right|^{L}}=1-\frac{\left|\Omega_{\mathcal{F}_{r}}\right|^{L}}{\left|\Omega_{\mathcal{F}_{i}}\right|^{L}} \leq 1-\frac{\left|\Omega_{\mathcal{F}_{r}}\right|^{L}}{\left|\Omega_{\mathcal{F}_{1}}\right|^{L}}=1-(1-\eta)^{L} \leq L \eta .
$$

Suppose the equivalent-structure learning algorithm $\mathcal{A}$ has success probability at least $1 / r+\alpha$, then

Hence,

$$
\operatorname{Pr}\left[\mathcal{A}(\Gamma) \in \mathcal{F}_{i}\right] \geq \frac{1}{r}+\alpha
$$

$$
\frac{1}{r}+\alpha \leq \frac{1}{\left|\Omega_{\mathcal{F}_{r}}\right|^{L}} \sum_{x \in\left(\Omega_{\mathcal{F}_{r}}\right)^{L}} \operatorname{Pr}\left[\mathcal{A}(x) \in \mathcal{F}_{i}\right]+L \eta
$$

Since $\sum_{i=1}^{r} \operatorname{Pr}\left[\mathcal{A}(x) \in \mathcal{F}_{i}\right]=1$ for any fixed sample sequence $x$, summing up over $i$ we get

$$
1+r \alpha \leq \frac{1}{\left|\Omega_{\mathcal{F}_{r}}\right|^{L}} \sum_{x \in\left(\Omega_{\mathcal{F}_{r}}\right)^{L}} \sum_{i=1}^{r} \operatorname{Pr}\left[\mathcal{A}(x) \in \mathcal{F}_{i}\right]+r L \eta=1+r L \eta .
$$

Hence, $L \geq \alpha / \eta$ as claimed.

Combining the results from Sections 3.2,3.3, and 3.4, we obtain the proof of Theorem 1.4: part (1) follows from Lemmas 3.1 and 3.2, part (2) follows from Theorem 3.3, and Corollary 3.8 implies part (3) of the theorem.

\subsection{General Lower Bound for Structure Learning of $q$-Colorings}

When $d-\sqrt{d}+\Theta(1)<q \leq d$ (more specifically, $q \leq d<q+[\sqrt{q}]-1$ ), structure learning for $q$ colorings is not identifiable, and the strong lower bound from part (3) of Theorem 3.3 (i.e., Corollary 3.8) does not apply either. In this section, we establish a weaker but more general lower bound for proper colorings that applies in this regime. Specifically, we provide a family of graphs $\mathcal{F} \subseteq$ $\mathcal{G}(n, d)$ such that the number of random $q$-colorings required to learn any graph in $\mathcal{F}$ with success probability at least $1 / 2$ is $\exp (\Omega(d-q))$.

Theorem 3.9. Let $d, q, n \in \mathbb{N}^{+}$such that $3 \leq q \leq d$ and $n \geq d+2$. Then, any equivalent-structure learning algorithm for $\mathcal{G}(n, d)$ with success probability at least $1 / 2$ requires at least $\exp (\Omega(d-q))$ samples.

Let $d, q, n \in \mathbb{N}^{+}$such that $3 \leq q \leq d$ and $n \geq d+2$. Let $C$ be a clique of size $q-3$, let $I$ be an independent set of size $d-q+1$, and let $W=\left\{w_{1}, \ldots, w_{n-d-1}\right\}$ be a simple path. In addition, let $u, v, w$ be three additional vertices that are not in $C, I$, or $W$. Define the graph $G=(V, E)$ such that

$$
V=V(C) \cup V(I) \cup\left\{w_{1}, \ldots, w_{n-d-1}\right\} \cup\{u, v, w\},
$$




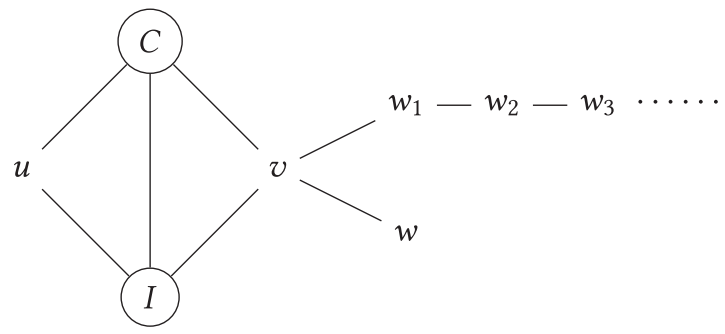

Fig. 4. The graph $G$ where $C=K_{q-3}$ and $I$ is an independent set of size $d-q+1$.

where $V(C)$ and $V(I)$ are the vertices in $C$ and $I$, respectively. In addition to the edges in $C$ and $W$, $G$ has the following edges:

(1) every vertex in $C$ is adjacent to every vertex in $I$;

(2) $u$ and $v$ are adjacent to every vertex in $C$ and $I$;

(3) $w, w_{1}$ are adjacent to $v$;

see Figure 4.

Let

$$
\mathcal{G}=\left\{G_{1}=G, G_{2}=(V, E \cup\{u w\}), G_{3}=\left(V, E \cup\left\{u w_{1}\right\}\right), G_{4}=\left(V, E \cup\left\{u w, u w_{1}\right\}\right)\right\} .
$$

Note that every graph in $\mathcal{G}$ is an $n$-vertex graph of maximum degree at most $d$, and thus $\mathcal{G} \subseteq$ $\mathcal{G}(n, d)$. Furthermore, for $1 \leq i \leq 4$, let $\mathcal{F}_{i}$ be the family of all graphs in $\mathcal{G}(n, d)$ that have the same set of $q$-colorings as $G_{i}$, and let $\mathcal{F}=\bigcup_{i=1}^{4} \mathcal{F}_{i}$. The following theorem immediately implies Theorem 3.9.

Theorem 3.10. Let $d, q, n \in \mathbb{N}^{+}$such that $3 \leq q \leq d$ and $n \geq d+2$. Then the number of independent random q-colorings required to learn any graph in $\mathcal{F}$ with probability at least $1 / 2$ is $\exp (\Omega(d-q))$.

Proof. If $u$ and $v$ receive distinct colors in a $q$-coloring of $G$, then the clique $C$ will be colored by $q-3$ of the $q-2$ colors not used by $u$ and $v$ and the number of available colors for every vertex in $I$ is only 1 . Thus, the number of colorings of $G$ where $u$ and $v$ receive distinct colors is $q !(q-1)^{n-d}$. Otherwise, if $u$ and $v$ receive the same color in a coloring of $G$, then $C$ will use $q-3$ of the $q-1$ available colors and every vertex in $I$ has 2 available color choices. Hence, the number of such colorings is $q !(q-1)^{n-d} \cdot 2^{d-q}$. Any $q$-coloring of $G$ where $u$ and $v$ receive the same color is also a proper $q$-coloring of $G_{4}$. Therefore, we get

$$
\frac{\left|\Omega_{\mathcal{F}_{4}}\right|}{\left|\Omega_{\mathcal{F}_{1}}\right|}=\frac{\left|\Omega_{G_{4}}\right|}{\left|\Omega_{G}\right|} \geq \frac{q !(q-1)^{n-d} \cdot 2^{d-q}}{q !(q-1)^{n-d}+q !(q-1)^{n-d} \cdot 2^{d-q}}=1-\mathrm{e}^{-\Omega(d-q)} .
$$

Thus, it follows from Lemma 3.7 that any equivalent-structure learning algorithm for $\mathcal{F}$ that succeeds with probability at least $1 / 2$ requires

$$
L \geq\left(\frac{1}{2}-\frac{1}{4}\right)\left(1-\frac{\left|\Omega_{\mathcal{F}_{4}}\right|}{\left|\Omega_{\mathcal{F}_{1}}\right|}\right)^{-1}=\mathrm{e}^{\Omega(d-q)}
$$

samples.

Theorem 3.9 follows immediately from Theorem 3.10 and the fact that $\mathcal{F} \subseteq \mathcal{G}(n, d)$. 


\section{LEARNING $\boldsymbol{H}$-colorings IN THE UNIQUENESS REGION}

As mentioned in Section 1, our results in Section 3 for statistical identifiability, structure learning, and equivalent-structure learning for proper colorings reveal a tight connection between the computational hardness of these problems and the uniqueness/non-uniqueness phase transition; see Georgii [25] for the definition of Gibbs uniqueness. In this section, we explore this connection.

Definition 4.1. Let $H$ be an arbitrary constraint graph, and let $G=(V, E)$ be an $H$-colorable graph. For $w \in V$, let

$$
S_{w}:=\left\{\left(\tau, \tau_{w}\right): \tau, \tau_{w} \in\{1, \ldots, q\}^{|V|} \text { and } \tau(z)=\tau_{w}(z) \forall z \neq w\right\} .
$$

For $v, w \in V$, let

$$
R_{v, w}:=\max _{\left(\tau, \tau_{w}\right) \in S_{w}}\left\|\pi_{v}(\cdot \mid \tau(\partial v))-\pi_{v}\left(\cdot \mid \tau_{w}(\partial v)\right)\right\|_{\mathrm{TV}},
$$

where $\pi_{v}(\cdot \mid \tau(\partial v))$ and $\pi_{v}\left(\cdot \mid \tau_{w}(\partial v)\right)$ are the conditional distributions at $v$ given the respective assignments $\tau$ and $\tau_{w}$ on the neighbors of $v$. Let $\alpha:=\max _{w \in V} \sum_{v \in \partial w} R_{v, w}$.

When $\alpha<1$, which is when the total influence of a site is strictly less than 1, the Gibbs distribution $\pi_{G}$ is known to be unique $[16,48]$.

Remark 4.2. Dobrushin and Shlosman [16] were the first to consider the maximum total influence of a site as a criteria for the uniqueness of the Gibbs measure. Their framework is slightly different: they focus on the infinite integer lattice graph $\mathbb{Z}^{d}$ and consider the influence of a site on constant size regions (not only single vertices). The Dobrushin-Shlosman condition was extended to general graphs in Weitz [48]. The condition we use here $(\alpha<1)$, which may be viewed as a special case of the (generalized) Dobrushin-Shlosman condition in Weitz [48], appeared before in Dyer et al. [18]. We note that the standard Dobrushin uniqueness condition [15] corresponds to the maximum row sum of $R$ being strictly less than 1; see Dyer et al. [18] for further discussion.

When $\alpha<1$, we deduce the following key property.

Lemma 4.3. Let $H \neq K_{q}^{+}$be an arbitrary constraint graph, and suppose $\{i, j\} \notin E(H)$. Let $G=(V, E)$ be a graph such that the uniqueness condition $\alpha<1$ is satisfied for $\pi_{G}$. Then, for all $\{u, v\} \notin E$,

$$
\operatorname{Pr}\left[X_{u}=i, X_{v}=j\right] \geq \frac{(1-\alpha)^{2}}{q^{2}} .
$$

Lemmas 4.3 and 3.1 imply that the STRUCTLEARN-H algorithm requires $L=O\left(q^{2} \log \left(\frac{n^{2}}{\varepsilon}\right)\right)$ independent samples to succeed with probability at least $1-\varepsilon$ and has running time is $O\left(\operatorname{Ln}^{2}\right)$. This establishes Theorem 1.5 from Section 1.

Proof of Lemma 4.3. For $u \in V$, we show first that $\operatorname{Pr}\left[X_{u}=i\right] \geq(1-\alpha) / q$. If $\sigma$ is an $H$-coloring of $G$ sampled according to $\pi_{G}$, we may update the color of any vertex $w \in V$ by choosing a new color for $w$ uniformly at random among the available colors for $w$ given $\sigma(V \backslash w)$. The resulting $H$-coloring after this update has distribution $\pi_{G}$.

Suppose $\sigma_{0}$ is an $H$-coloring of $G$ sampled according to $\pi_{G}$, and let $\tau_{0}$ be the color assignment that agrees with $\sigma_{0}$ everywhere except possibly at $u$, where we set $\tau_{0}(u)=i$. (Note that $\tau_{0}$ is not necessarily a valid $H$-coloring.)

Let $\partial u=\left\{v_{1}, \ldots, v_{l}\right\}$. We update the configuration in $v_{1}$, then in $v_{2}$ and so on, in both $\sigma_{0}$ and $\tau_{0}$; then we update the color of $u$. Let $\sigma_{k}$ and $\tau_{k}$ be the configuration on $V \backslash u$ after updating $v_{k}$ in $\sigma_{k-1}$ and $\tau_{k-1}$, respectively. The color of $v_{k}$ in both $\sigma_{k-1}$ and $\tau_{k-1}$ is updated using the optimal coupling $v_{k}$ between the distributions $\pi_{v_{k}}\left(\cdot \mid \sigma_{k-1}\right)$ and $\pi_{v_{k}}\left(\cdot \mid \tau_{k-1}\right)$ as follows. Sample $\left(a_{k}, b_{k}\right)$ from $v_{k}$, and let 
$\sigma_{k}\left(V \backslash v_{k}\right)=\sigma_{k-1}\left(V \backslash v_{k}\right), \sigma_{k}\left(v_{k}\right)=a_{k}, \tau_{k}\left(V \backslash v_{k}\right)=\tau_{k-1}\left(V \backslash v_{k}\right)$ and $\tau\left(v_{k}\right)=b_{k}$. After updating $\partial u=\left\{v_{1}, \ldots, v_{l}\right\}$ in this manner, $\sigma_{l}$ has law $\pi_{G}$. Moreover,

$$
\begin{aligned}
\operatorname{Pr}\left[\sigma_{l} \neq \tau_{l}\right] & \leq \operatorname{Pr}_{v_{l}}\left[\sigma_{l} \neq \tau_{l} \mid \sigma_{l-1}=\tau_{l-1}\right]+\operatorname{Pr}\left[\sigma_{l-1} \neq \tau_{l-1}\right] \\
& \leq \sum_{k=1}^{l} \operatorname{Pr}_{v_{k}}\left[\sigma_{k} \neq \tau_{k} \mid \sigma_{k-1}=\tau_{k-1}\right] \\
& =\sum_{k=1}^{l}\left\|\pi_{v_{k}}\left(\cdot \mid \tau_{k-1}\left(V \backslash\left\{u, v_{k}\right\}\right), \sigma_{0}(u)\right)-\pi_{v_{k}}\left(\cdot \mid \tau_{k-1}\left(V \backslash\left\{u, v_{k}\right\}\right), \tau_{0}(u)\right)\right\|_{\mathrm{TV}} \\
& \leq \sum_{k=1}^{l} R_{v_{k}, u} \leq \alpha,
\end{aligned}
$$

where the last two inequalities follow from the definitions of $R_{v_{k}, u}$ and $\alpha$ (Definition 4.1). Hence, with probability at least $1-\alpha, \sigma_{l}=\tau_{l}$. If this is the case, then color $i$ is compatible with $\sigma_{l}(V \backslash u)$, and thus when $u$ is updated, it receives color $i$ with probability at least $1 / q$. Thus, we get

$$
\operatorname{Pr}\left[X_{u}=i\right] \geq \frac{(1-\alpha)}{q} .
$$

Finally, let $v \in V$ such that $v \notin \partial u$. Using the procedure described earlier to update the configuration in $\partial u \cup u$, and then in $\partial v \cup v$, we obtain

$$
\operatorname{Pr}\left[X_{u}=i, X_{v}=j\right] \geq \frac{(1-\alpha)^{2}}{q^{2}} .
$$

\section{APPROXIMATE-STRUCTURE LEARNING OF H-COLORINGS}

In addition to structure learning (exact recovery of the hidden graph $G$ ) and equivalent-structure learning (learning a graph with the same set of $H$-colorings), we may consider the corresponding approximation problem of finding a graph $\hat{G}$ such that $\pi_{\hat{G}}$ is close to $\pi_{G}$ in some notion of distance, such as total variation distance or Kullback-Leibler divergence. Apparently, this task is much simpler for hard-constraint systems.

In this section, we consider this approximation variant of structure learning for hard-constraint systems with respect to total variation distance. In other words, given $L$ independent samples $\sigma^{(1)}, \ldots, \sigma^{(L)}$ from $\pi_{G}$, we consider the problem of finding a graph $\hat{G}$ such that

$$
\left\|\pi_{G}-\pi_{\hat{G}}\right\|_{\mathrm{TV}}<\gamma
$$

where $\gamma>0$ is a desired precision.

Theorem 5.1. Suppose $H \neq K_{q}^{+}$, and let $\hat{G}$ be the output of the STRUCTLEARN-H algorithm. Then, for all $\varepsilon \in(0,1)$ and $\gamma \in(0,1)$,

$$
\operatorname{Pr}\left[\left\|\pi_{G}-\pi_{\hat{G}}\right\|_{T V}<\gamma\right] \geq 1-\varepsilon
$$

provided $L \geq(2 \gamma)^{-1} n^{2} \log \left(\frac{n^{2}}{2 \varepsilon}\right)$.

Recall that the running time of STRUCTLEARN-H is $O\left(L n^{2}\right)$, and thus from Theorem 5.1, we get an algorithm for approximate structure learning with running time $O\left(\gamma^{-1} n^{4} \log \left(\frac{n}{\varepsilon}\right)\right)$.

Proof of Theorem 5.1. Let $\hat{G}=(V(\hat{G}), E(\hat{G}))$. Recall that $\{u, v\} \notin E(\hat{G})$ if and only if $u, v$ receive incompatible colors in one of the samples $\sigma^{(1)}, \ldots, \sigma^{(L)}$ from $\pi_{G}$. Hence, $\hat{G}$ is a supergraph of $G$ 
and so $\Omega_{\hat{G}} \subseteq \Omega_{G}$. Moreover,

$$
\left\|\pi_{G}-\pi_{\hat{G}}\right\|_{\mathrm{TV}}=\sum_{\sigma \in \Omega_{G} \backslash \Omega_{\hat{G}}} \frac{1}{\left|\Omega_{G}\right|}=\frac{\left|\Omega_{G}\right|-\left|\Omega_{\hat{G}}\right|}{\left|\Omega_{G}\right|}=\operatorname{Pr}\left[\sigma \notin \Omega_{\hat{G}}\right],
$$

assuming $\sigma$ is an $H$-coloring of $G$ chosen uniformly at random (i.e., $\sigma$ is drawn from $\pi_{G}$ ). If we let $\Gamma=E(\hat{G}) \backslash E(G)$, then

$$
\operatorname{Pr}\left[\sigma \notin \Omega_{\hat{G}}\right]=\operatorname{Pr}\left[\exists\{u, v\} \in \Gamma:\left\{\sigma_{u}, \sigma_{v}\right\} \notin E(H)\right] \leq \sum_{\{u, v\} \in \Gamma} \operatorname{Pr}\left[\left\{\sigma_{u}, \sigma_{v}\right\} \notin E(H)\right]
$$

by a union bound.

Now, for $\gamma>0$, let

$$
M_{\gamma}=\left\{\{u, v\} \notin E(G): \operatorname{Pr}\left[\left\{\sigma_{u}, \sigma_{v}\right\} \notin E(H)\right] \geq \frac{2 \gamma}{n^{2}}\right\} .
$$

Let $Z_{u v}$ be the number of samples $\sigma^{(1)}, \ldots, \sigma^{(L)}$ where vertices $u$ and $v$ receive incompatible colors. Then for any $\{u, v\} \in M_{\gamma}$,

$$
\operatorname{Pr}\left[Z_{u v}=0\right] \leq\left(1-\frac{2 \gamma}{n^{2}}\right)^{L} \leq \exp \left(\frac{-2 \gamma L}{n^{2}}\right) \leq \frac{2 \varepsilon}{n^{2}} .
$$

A union bound then implies that with probability at least $1-\varepsilon$, all edges in $M_{\gamma}$ are not in $E(\hat{G})$. Hence, with probability at least $1-\varepsilon$, all edges in $E(\hat{G})$ satisfy:

$$
\operatorname{Pr}\left[\left\{\sigma_{u}, \sigma_{v}\right\} \notin E(H)\right]<\frac{2 \gamma}{n^{2}} .
$$

Plugging this bound into (5), we get

$$
\operatorname{Pr}\left[\left\|\pi_{G}-\pi_{\hat{G}}\right\|_{\mathrm{TV}}<\gamma\right] \geq 1-\varepsilon
$$

as desired.

\section{LEARNING WEIGHTED $H$-COLORINGS}

In this section, we consider the more general setting of weighted $H$-colorings. We restrict our attention to constraint graphs with at least one hard constraint, which corresponds to spin systems with hard constraints.

\subsection{Spin Systems with Hard Constraints}

Let $G=(V, E)$ be an undirected weighted graph with weights given by the function $\theta: E \cup V \rightarrow$ $\mathbb{R}^{+}$. (For definiteness, we only consider a positive weight function $\theta$.) A spins system on the graph $G$ consists of a set of spins $[q]=\{1, \ldots, q\}$, a symmetric edge potential $J:[q] \times[q] \rightarrow \mathbb{R} \cup\{-\infty\}$, and a vertex potential $h:[q] \rightarrow \mathbb{R}$. A configuration $\sigma: V \rightarrow[q]$ of the system is an assignment of spins to the vertices of $G$. Each configuration $\sigma \in[q]^{V}$ is assigned probability

$$
\pi_{G, \theta}(\sigma)=\frac{1}{Z_{G, \theta}} \exp \left(\sum_{\{u, v\} \in E} \theta(u, v) J\left(\sigma_{u}, \sigma_{v}\right)+\sum_{u \in V} \theta(u) h\left(\sigma_{u}\right)\right),
$$

where $Z_{G, \theta}$ is the normalizing constant called the partition function. If $J(i, j)=-\infty$ for some $i, j \in$ $[q]$, then $\{i, j\}$ is a hard constraint; otherwise, $i$ and $j$ are compatible. 
Unweighted $H$-colorings, which were considered in Sections 2, 3, and 4, correspond to the special case where $\theta=1, h=0$, and

$$
J(i, j)= \begin{cases}1 & \text { if }(i, j) \in E(H) \\ -\infty & \text { if }(i, j) \notin E(H) .\end{cases}
$$

In this section, we consider the structure learning problem for a class of models known as permissive systems. This is a widely used notion in statistical physics for spin systems with hard constraints (e.g., see [14, 20, 37]). There are several different notions in the literature, but here we consider the weakest one (i.e., the easiest to satisfy). Roughly, the condition says that for any boundary condition there is always a valid configuration for the interior.

Definition 6.1. A spin system is called permissive if for any $A \subseteq V$ and any valid configuration $\tau$ on $V \backslash A$, there is at least one valid configuration $\sigma$ on $A$ such that $\pi(\sigma \mid \tau)>0$.

Independent sets, and more generally the hard-core model, are examples of permissive models since we can assign spin 0 (unoccupied) to the vertices in $A$.

\subsection{Structure Learning for Spin Systems with Hard Constraints}

We first formalize the notion of structure learning for the setting of weighted constraint graphs. Suppose we know the number of spins $q$, the edge potential $J \in \mathbb{R}^{q \times q}$, and the vertex potential $h \in \mathbb{R}^{q}$ of a spin system $\mathcal{S}$. Consider the family of graphs

$$
\begin{aligned}
\mathcal{G}(n, d, \beta, \gamma)=\{(G=(V, E), \theta): & |V|=n, \\
& \Delta(G) \leq d, \\
& |\theta(u, v)| \leq \beta \text { for all }\{u, v\} \in E, \\
& |\theta(v)| \leq \gamma \text { for all } v \in V\},
\end{aligned}
$$

where $\Delta(G)$ denotes the maximum degree of the graph $G$. Suppose that we are given $L$ independent samples $\sigma^{(1)}, \sigma^{(2)}, \ldots, \sigma^{(L)}$ from the distribution $\pi_{G, \theta}$ where $(G, \theta) \in \mathcal{G}(n, d, \beta, \gamma)$. A structure learning algorithm for the spin system $\mathcal{S}$ and the family $\mathcal{G}(n, d, \beta, \gamma)$ takes as input the sample sequence $\sigma^{(1)}, \sigma^{(2)}, \ldots, \sigma^{(L)}$ and outputs an estimator $\hat{G}$ such that $\operatorname{Pr}[G=\hat{G}] \geq 1-\varepsilon$, where $\varepsilon>0$ is a prescribed failure probability.

\subsection{Learning Permissive Spin Systems}

In this section, we analyze the running time and sample complexity of the STRUCTLEARN-H algorithm for permissive spin systems.

Let $\hat{\gamma}=\gamma \cdot \max _{i \in[q]}|h(i)|$ and $\hat{\beta}=\beta \cdot \max _{i, j \in[q]}|J(i, j)|$. Recall that for $v \in V, X_{v}$ denotes the random variable for the color of $v$ under $\pi_{G}$. We show that for permissive systems, the running time of STRUCTLEARN-H is polynomial in the size of the graph but depends exponentially on $\hat{\gamma}, \hat{\beta}$ and its maximum degree.

Theorem 6.2. Let $(G, \theta) \in \mathcal{G}(n, d, \beta, \gamma)$, and suppose that $\mathcal{S}$ is a permissive spin system with at least one hard constraint. Then, for all $\varepsilon \in(0,1)$, if the STRUCTLEARN-H algorithm receives as input

$$
L \geq q^{2(d+1)} \exp (4(\hat{\beta} d+\hat{\gamma})(d+1)) \log \left(\frac{n^{2}}{2 \varepsilon}\right)
$$

independent samples from $\pi_{G, \theta}$, it outputs the graph $G$ with probability at least $1-\varepsilon$ and has running time $O\left(L n^{2}\right)$.

Theorem 6.2 yields a structure learning algorithm for the hard-core model for all $\lambda>0$; thus, it generalizes the algorithmic result of Bresler et al. [6]. We observe also that the running time of 
our algorithm for permissive systems is comparable to the running time of the optimal structure learning algorithms for soft-constraint systems in Klivans and Meka [33].

Theorem 6.2 is a direct corollary of the following lemma and Lemma 3.1.

Lemma 6.3. Suppose that $\mathcal{S}$ is a permissive spin system with at least one hard constraint $\{i, j\} \in$ $[q] \times[q]$ on a weighted graph $(G, \theta) \in \mathcal{G}(n, d, \beta, \gamma)$. Then, for all $\{u, v\} \notin E$,

$$
\operatorname{Pr}\left[X_{u}=i, X_{v}=j\right] \geq q^{-2(d+1)} \exp (-4(\hat{\beta} d+\hat{\gamma})(d+1)) .
$$

In the proof of Lemma 6.3, we use the following fact.

FACT 6.4. Let $R \subseteq V$, and let $\tau$ be a configuration on $\partial R$. If $\Omega^{\tau}(R) \neq \emptyset$ is the set of valid configurations on $R$ given $\tau$, then for any $\sigma \in \Omega^{\tau}(R)$,

$$
\operatorname{Pr}\left[X_{R}=\sigma \mid X_{\partial R}=\tau\right] \geq q^{-|R|} \exp (-2(\hat{\beta} d+\hat{\gamma})|R|) .
$$

We are now ready to prove Lemma 6.3.

Proof of Lemma 6.3. For any $A \subseteq V$ and any spin configuration $\sigma$ of $A$, with a slight abuse of notation we use $\{\sigma\}$ for the event $\left\{X_{A}=\sigma\right\}$.

Let $u, v \in V$ such that $\{u, v\} \notin E$, and let $N_{1}$ and $N_{2}$ be the set of vertices at distances one and two, respectively, from $\{u, v\}$ (i.e., $N_{1}=\partial u \cup \partial v$ and $N_{2}=\left\{w \in \partial N_{1}: w \neq u, w \neq v\right\}$ ). Let $\Omega_{1}$ and $\Omega_{2}$ be the set of valid configurations for $N_{1}$ and $N_{2}$, respectively. Then,

$$
\operatorname{Pr}\left[X_{u}=i, X_{v}=j\right] \geq \min _{\tau_{2} \in \Omega_{2}} \operatorname{Pr}\left[X_{u}=i, X_{v}=j \mid \tau_{2}\right] .
$$

Since the spin system is permissive, for any $\tau_{2} \in \Omega_{2}$ there exists $\tau_{1} \in \Omega_{1}$ such that

$$
\operatorname{Pr}\left[\tau_{1} \mid \tau_{2}, X_{u}=i, X_{v}=j\right]>0 .
$$

Then,

$$
\operatorname{Pr}\left[X_{u}=i, X_{v}=j \mid \tau_{2}\right] \geq \operatorname{Pr}\left[X_{u}=i, X_{v}=j \mid \tau_{1}\right] \operatorname{Pr}\left[\tau_{1} \mid \tau_{2}\right] \geq q^{-2} \mathrm{e}^{-4(\hat{\beta} d+\hat{\gamma})} \operatorname{Pr}\left[\tau_{1} \mid \tau_{2}\right],
$$

by Fact 6.4 . Now,

$$
\operatorname{Pr}\left[\tau_{1} \mid \tau_{2}\right]=\sum_{a, b \in[q]} \operatorname{Pr}\left[\tau_{1} \mid X_{u}=a, X_{v}=b, \tau_{2}\right] \operatorname{Pr}\left[X_{u}=a, X_{v}=b \mid \tau_{2}\right] .
$$

Since $\left|N_{1}\right| \leq 2 d$, by Fact $6.4, \operatorname{Pr}\left[\tau_{1} \mid X_{u}=a, X_{v}=b, \tau_{2}\right] \geq q^{-2 d} \exp (-4(\hat{\beta} d+\hat{\gamma}) d)$. Together with (7) and (8), this implies

$$
\operatorname{Pr}\left[X_{u}=i, X_{v}=j\right] \geq q^{-2(d+1)} \exp (-4(\hat{\beta} d+\hat{\gamma})(d+1)) .
$$

Remark 6.5. A simplified version of this argument can be used to show that in a permissive $H$-coloring, for any hard constraint $\{i, j\} \notin E(H), \operatorname{Pr}\left[X_{u}=i, X_{v}=j\right] \geq 1 / q^{2 d}$. From this we obtain a structure learning algorithm for permissive $H$-colorings with running time $O\left(q^{2 d} n^{2} \log n\right)$ via Lemma 3.1.

We conclude this section with the proof of Fact 6.4.

Proof of Fact 6.4. For $\sigma \in \Omega^{\tau}(R)$, let

$$
w(\sigma)=\exp \left[\sum_{u \in R} \sum_{v \in \partial u \cap R} \theta(u, v) J\left(\sigma_{u}, \sigma_{v}\right)+\sum_{u \in R} \sum_{v \in \partial u \cap \partial R} \theta(u, v) J\left(\sigma_{u}, \tau_{v}\right)+\sum_{u \in R} \theta(u) h\left(\sigma_{u}\right)\right] .
$$

Then,

$$
\operatorname{Pr}\left[X_{R}=\sigma \mid X_{\partial R}=\tau\right]=\frac{w(\sigma)}{Z_{R}^{\tau}}
$$


with $Z_{R}^{\tau}=\sum_{\sigma^{\prime} \in \Omega^{\tau}(R)} w\left(\sigma^{\prime}\right)$. Observe that for all $\sigma^{\prime} \in \Omega^{\tau}(R), \exp (-(\hat{\beta} d+\hat{\gamma})|R|) \leq w\left(\sigma^{\prime}\right) \leq$ $\exp ((\hat{\beta} d+\hat{\gamma})|R|)$. Hence, $Z_{R}^{\tau} \leq q^{|R|} \exp ((\hat{\beta} d+\hat{\gamma})|R|)$ and

$$
\operatorname{Pr}\left[X_{R}=\sigma \mid X_{\partial R}=\tau\right] \geq q^{-|R|} \exp (-2(\hat{\beta} d+\hat{\gamma})|R|) .
$$

\subsection{Identifiability for Weighted $H$-Colorings}

We prove next an analog of our characterization theorem (Theorem 1.2) for identifiability of weighted $H$-colorings. The edge potential $J$ corresponds to the weighted adjacency matrix of a weighted constraint graph $H^{J}=\left(V\left(H^{J}\right), E\left(H^{J}\right)\right)$, where $V\left(H^{J}\right)=\{1, \ldots, q\},\{i, j\} \notin E\left(H^{J}\right)$ if and only if $J(i, j)=-\infty$, and the weight of $\{i, j\} \in E\left(H^{J}\right)$ is $J(i, j)$. As before, we say that a graph $G$ is $H^{J}$-colorable if there is a valid $H^{J}$-coloring for $G$. If $\{i, j\} \notin E\left(H^{J}\right)$, we call $\{i, j\}$ a hard constraint. The notion of identifiability extends to the weighted setting as follows.

Definition 6.6. A weighted constraint graph $H^{J}$ is said to be identifiable with respect to a family of $H^{J}$-colorable graphs $\mathcal{G}$ if for any two distinct graphs $G_{1}, G_{2} \in \mathcal{G}$ we have $\pi_{G_{1}} \neq \pi_{G_{2}}$. In particular, when $\mathcal{G}$ is the set of all finite $H^{J}$-colorable graphs, we say that $H^{J}$ is identifiable.

(Definition 1.1 is the analog definition in the unweighted setting.)

In our characterization theorem, we consider the supergraphs $G_{i j}$ 's introduced in the unweighted setting; see Definition 2.2 and Figure 1.

THEOREM 6.7. Let $H^{J}$ be a weighted constraint graph with at least one hard constraint. If $H^{J}$ has a self-loop, then $H^{J}$ is identifiable. Otherwise, $H^{J}$ is identifiable if and only if for each $\{i, j\} \in E\left(H^{J}\right)$ there exists an $H^{J}$-coloring $\sigma$ of $G_{i j}$ such that

$$
J\left(\sigma_{i}, \sigma_{j}\right)+J\left(\sigma_{i^{\prime}}, \sigma_{j^{\prime}}\right) \neq J\left(\sigma_{i^{\prime}}, \sigma_{j}\right)+J\left(\sigma_{i}, \sigma_{j^{\prime}}\right) .
$$

(Recall that $i^{\prime}$ and $j^{\prime}$ are the copies of the vertices $i$ and $j$ in $G_{i j}$.)

Proof. For clarity, we shall assume in this proof that the underlying graph $G=(V, E)$ is unweighted and that there is no external field (i.e., $\theta=1$ and $h=0$ ). The same proof generalizes to spin systems on weighted graphs with external field.

Henceforth, we use $H$ for $H^{J}$ to simplify the notation. We consider first the case when $H$ has no self-loops. For the forward direction, we consider the contrapositive. Suppose that there exists $\{i, j\} \in E(H)$ such that for every proper $H$-coloring $\sigma$ of $G_{i j}$ we have

$$
J\left(\sigma_{i}, \sigma_{j}\right)+J\left(\sigma_{i^{\prime}}, \sigma_{j^{\prime}}\right)=J\left(\sigma_{i^{\prime}}, \sigma_{j}\right)+J\left(\sigma_{i}, \sigma_{j^{\prime}}\right) .
$$

Under this assumption, we construct two distinct $H$-colorable graphs $F_{1}, F_{2}$ such that $\pi_{F_{1}}=\pi_{F_{2}}$; this implies that $H$ is not identifiable, which would complete the proof of the forward direction. For this, for each $\{i, j\} \in E(H)$, let us define the supergraph $\widetilde{G}_{i j}$ of $H$ that is the result of creating two copies $i^{\prime}, i^{\prime \prime}$ of vertex $i$ and two copies $j^{\prime}, j^{\prime \prime}$ of vertex $j$, with no edges between $i^{\prime}, i^{\prime \prime}, j^{\prime}, j^{\prime \prime}$. Formally, for each $\{i, j\} \in E(H)$, we define the graph $\widetilde{G}_{i j}=\left(V\left(\widetilde{G}_{i j}\right), E\left(\widetilde{G}_{i j}\right)\right)$ as follows:

(1) $V\left(G_{i j}\right)=V(H) \cup\left\{i^{\prime}, i^{\prime \prime}, j^{\prime}, j^{\prime \prime}\right\}$, where $i^{\prime}, i^{\prime \prime}, j^{\prime}, j^{\prime \prime}$ are four new colors;

(2) If $\{a, b\} \in E(H)$, then the edge $\{a, b\}$ is also in $E\left(\widetilde{G}_{i j}\right)$;

(3) For each $k \in V\left(\widetilde{G}_{i j}\right) \backslash\left\{i^{\prime}, i^{\prime \prime}, j^{\prime}, j^{\prime \prime}\right\}$, the edges $\left\{i^{\prime}, k\right\}$ and $\left\{i^{\prime \prime}, k\right\}$ are in $\widetilde{G}_{i j}$ if and only if the edge $\{i, k\}$ is in $H$, and similarly $\left\{j^{\prime}, k\right\},\left\{j^{\prime \prime}, k\right\} \in E\left(\widetilde{G}_{i j}\right)$ if and only if $\{j, k\} \in E(H)$;

see Figure 5 for an example.

Let $\sigma$ be an $H$-coloring of $\widetilde{G}_{i j}$. Since the subgraphs induced by $V\left(\widetilde{G}_{i j}\right) \backslash\left\{i^{*}, j^{*}\right\}$ with $i^{*} \in\left\{i^{\prime}, i^{\prime \prime}\right\}$ and $j^{*}=\left\{j^{\prime}, j^{\prime \prime}\right\}$ are all isomorphic to $G_{i j}$, our assumption implies

$$
J\left(\sigma_{i}, \sigma_{j}\right)+J\left(\sigma_{i^{\prime}}, \sigma_{j^{\prime}}\right)=J\left(\sigma_{i^{\prime}}, \sigma_{j}\right)+J\left(\sigma_{i}, \sigma_{j^{\prime}}\right),
$$




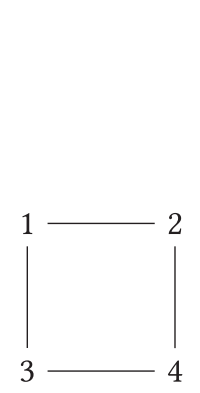

$H$

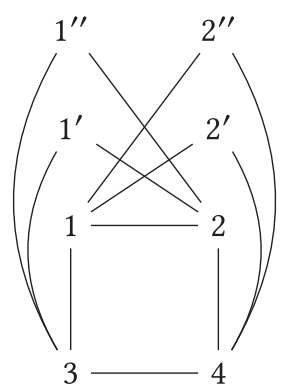

$G_{12}^{2}$

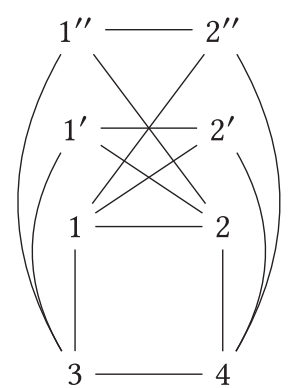

$F_{1}$

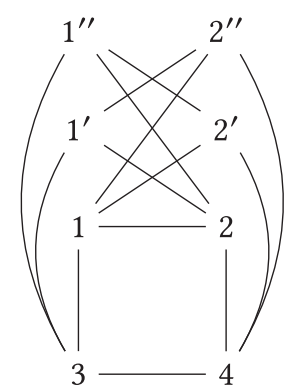

$F_{2}$

Fig. 5. A constraint graph $H$, its supergraph $\widetilde{G}_{12}$, and the graphs $F_{1}$ and $F_{2}$.

$$
\begin{gathered}
J\left(\sigma_{i}, \sigma_{j}\right)+J\left(\sigma_{i^{\prime \prime}}, \sigma_{j^{\prime \prime}}\right)=J\left(\sigma_{i^{\prime \prime}}, \sigma_{j}\right)+J\left(\sigma_{i}, \sigma_{j^{\prime \prime}}\right), \\
J\left(\sigma_{i}, \sigma_{j}\right)+J\left(\sigma_{i^{\prime \prime}}, \sigma_{j^{\prime}}\right)=J\left(\sigma_{i^{\prime \prime}}, \sigma_{j}\right)+J\left(\sigma_{i}, \sigma_{j^{\prime}}\right), \\
J\left(\sigma_{i}, \sigma_{j}\right)+J\left(\sigma_{i^{\prime}}, \sigma_{j^{\prime \prime}}\right)=J\left(\sigma_{i^{\prime}}, \sigma_{j}\right)+J\left(\sigma_{i}, \sigma_{j^{\prime \prime}}\right) .
\end{gathered}
$$

Since the sum of the right-hand sides of (9) and (10) is equal to the sum of the right-hand sides of (11) and (12), we get

$$
J\left(\sigma_{i^{\prime}}, \sigma_{j^{\prime}}\right)+J\left(\sigma_{i^{\prime \prime}}, \sigma_{j^{\prime \prime}}\right)=J\left(\sigma_{i^{\prime \prime}}, \sigma_{j^{\prime}}\right)+J\left(\sigma_{i^{\prime}}, \sigma_{j^{\prime \prime}}\right) .
$$

Now, let

$$
\begin{aligned}
& F_{1}=\left(V\left(\widetilde{G}_{i j}\right), E\left(\widetilde{G}_{i j}\right) \cup\left\{\left\{i^{\prime}, j^{\prime}\right\},\left\{i^{\prime \prime}, j^{\prime \prime}\right\}\right\}\right), \\
& F_{2}=\left(V\left(\widetilde{G}_{i j}\right), E\left(\widetilde{G}_{i j}\right) \cup\left\{\left\{i^{\prime \prime}, j^{\prime}\right\},\left\{i^{\prime}, j^{\prime \prime}\right\}\right\}\right) ;
\end{aligned}
$$

see Figure 5 . Then, using (6), for any $H$-coloring $\sigma$ of $\widetilde{G}_{i j}$, we have

$$
\frac{\pi_{F_{1}}(\sigma)}{\pi_{F_{2}}(\sigma)}=\frac{Z_{F_{1}}^{-1} \exp \left(J\left(\sigma_{i^{\prime}}, \sigma_{j^{\prime}}\right)+J\left(\sigma_{i^{\prime \prime}}, \sigma_{j^{\prime \prime}}\right)\right)}{Z_{F_{2}}^{-1} \exp \left(J\left(\sigma_{i^{\prime \prime}}, \sigma_{j^{\prime}}\right)+J\left(\sigma_{i^{\prime}}, \sigma_{j^{\prime \prime}}\right)\right)}=\frac{Z_{F_{2}}}{Z_{F_{1}}},
$$

which is a constant independent of $\sigma$. By (13), $\pi_{F_{1}}$ and $\pi_{F_{2}}$ have the same support. Moreover, any $H$-coloring of $F_{1}$ or $F_{2}$ is also an $H$-coloring of $\widetilde{G}_{i j}$. Hence, we conclude that $\pi_{F_{1}}=\pi_{F_{2}}$, implying $H$ is not identifiable. This completes the proof of the forward direction.

For the reverse direction, suppose that for all $\{i, j\} \in E(H)$ there exists an $H$-coloring $\sigma$ of $G_{i j}$ where

$$
J\left(\sigma_{i}, \sigma_{j}\right)+J\left(\sigma_{i^{\prime}}, \sigma_{j^{\prime}}\right) \neq J\left(\sigma_{i^{\prime}}, \sigma_{j}\right)+J\left(\sigma_{i}, \sigma_{j^{\prime}}\right) .
$$

Consider two $H$-colorable graphs $G_{1}=\left(V, E_{1}\right)$ and $G_{2}=\left(V, E_{2}\right)$ such that $\pi_{G_{1}}=\pi_{G_{2}}$. We show that for any $u, v \in V,\{u, v\} \in E_{1}$ if and only if $\{u, v\} \in E_{2}$, and thus $G_{1}=G_{2}$. This implies that $H$ is identifiable.

Let $u, v \in V$, and let $\tau$ be an $H$-coloring of $G_{1}$. Since $\pi_{G_{1}}=\pi_{G_{2}}$, then $\tau$ is also an $H$-coloring of $G_{2}$. Suppose $i=\tau(u)$ and $j=\tau(v)$. If $i$ and $j$ are not compatible, then $\{u, v\} \notin E_{1}$ and $\{u, v\} \notin E_{2}$. Thus, let us assume $i$ and $j$ are compatible. Let $\sigma$ be an $H$-coloring of $G_{i j}$ such that

$$
J(a, b)+J\left(a^{\prime}, b^{\prime}\right) \neq J\left(a^{\prime}, b\right)+J\left(a, b^{\prime}\right),
$$

where $a=\sigma_{i}, b=\sigma_{j}, a^{\prime}=\sigma_{i^{\prime}}, b^{\prime}=\sigma_{j^{\prime}}$; we know such an $H$-coloring exists by assumption. 
Now consider the conditional distribution $\mu$ on the vertices $u$ and $v$ of $G_{1}$ given the configuration $\tau(V \backslash\{u, v\})$. Then,

$$
\begin{aligned}
& p_{1}\left(G_{1}\right):=\mu\left(X_{u}=a, X_{v}=b\right)=\frac{1}{Z_{\text {cond }}\left(G_{1}\right)} \exp \left[h_{a}+h_{b}+\mathbb{1}\left(\{u, v\} \in E_{1}\right) J(a, b)\right] \\
& p_{2}\left(G_{1}\right):=\mu\left(X_{u}=a^{\prime}, X_{v}=b^{\prime}\right)=\frac{1}{Z_{\text {cond }}\left(G_{1}\right)} \exp \left[h_{a^{\prime}}+h_{b^{\prime}}+\mathbb{1}\left(\{u, v\} \in E_{1}\right) J\left(a^{\prime}, b^{\prime}\right)\right] \\
& p_{3}\left(G_{1}\right):=\mu\left(X_{u}=a^{\prime}, X_{v}=b\right)=\frac{1}{Z_{\text {cond }}\left(G_{1}\right)} \exp \left[h_{a^{\prime}}+h_{b}+\mathbb{1}\left(\{u, v\} \in E_{1}\right) J\left(a^{\prime}, b\right)\right] \\
& p_{4}\left(G_{1}\right):=\mu\left(X_{u}=a, X_{v}=b^{\prime}\right)=\frac{1}{Z_{\text {cond }}\left(G_{1}\right)} \exp \left[h_{a}+h_{b^{\prime}}+\mathbb{1}\left(\{u, v\} \in E_{1}\right) J\left(a, b^{\prime}\right)\right],
\end{aligned}
$$

where $Z_{\text {cond }}\left(G_{1}\right)$ is the normalizing factor for $\mu$,

$$
\begin{aligned}
& h_{a}=\sum_{w \in \partial u} J(a, \tau(w)), \\
& h_{b}=\sum_{w \in \partial v} J(b, \tau(w))
\end{aligned}
$$

and $h_{a^{\prime}}, h_{b^{\prime}}$ are defined in similar manner. This gives

$$
\frac{p_{1}\left(G_{1}\right) p_{2}\left(G_{1}\right)}{p_{3}\left(G_{1}\right) p_{4}\left(G_{1}\right)}=\exp \left[\mathbb{1}\left(\{u, v\} \in E_{1}\right)\left(J(a, b)+J\left(a^{\prime}, b^{\prime}\right)-J\left(a^{\prime}, b\right)-J\left(a, b^{\prime}\right)\right)\right] .
$$

Since by assumption $J(a, b)+J\left(a^{\prime}, b^{\prime}\right)-J\left(a^{\prime}, b\right)-J\left(a, b^{\prime}\right) \neq 0,\{u, v\} \in E\left(G_{1}\right)$ if and only if $p_{1}\left(G_{1}\right) p_{2}\left(G_{1}\right) \neq p_{3}\left(G_{1}\right) p_{4}\left(G_{1}\right)$. Moreover, $\pi_{G_{1}}=\pi_{G_{2}}$, and thus $p_{k}\left(G_{1}\right)=p_{k}\left(G_{2}\right)$ for $k \in\{1,2,3,4\}$. Hence,

$$
\frac{p_{1}\left(G_{1}\right) p_{2}\left(G_{1}\right)}{p_{3}\left(G_{1}\right) p_{4}\left(G_{1}\right)}=\frac{p_{1}\left(G_{2}\right) p_{2}\left(G_{2}\right)}{p_{3}\left(G_{2}\right) p_{4}\left(G_{2}\right)} .
$$

This implies that $\{u, v\} \in E_{1}$ if and only if $\{u, v\} \in E_{2}$, and thus $G_{1}=G_{2}$. This completes the proof of the reverse direction when $H$ does not have self-loops. When $H$ has at least one self-loop, then using the argument in the proof of Lemma 2.3, which generalizes straightforwardly to the weighted setting, we get that $H$ is identifiable.

\section{REFERENCES}

[1] Pieter Abbeel, Daphne Koller, and Andrew Y. Ng. 2006. Learning factor graphs in polynomial time and sample complexity. Journal of Machine Learning Research 7 (Aug. 2006), 1743-1788.

[2] Anima Anandkumar, Daniel J. Hsu, Furong Huang, and Sham M. Kakade. 2012. Learning mixtures of tree graphical models. In Advances in Neural Information Processing Systems (NeurIPS'12). 1052-1060.

[3] Ivona Bezáková, Antonio Blanca, Zongchen Chen, Daniel Štefankovič, and Eric Vigoda. 2019. Lower bounds for testing graphical models: Colorings and antiferromagnetic Ising models. In Proceedings of the 31st Conference on Learning Theory (COLT'19). 283-298.

[4] Guy Bresler. 2015. Efficiently learning Ising models on arbitrary graphs. In Proceedings of the 47th Annual ACM Symposium on Theory of Computing (STOC'15). ACM, New York, NY, 771-782.

[5] Guy Bresler, David Gamarnik, and Devavrat Shah. 2014. Hardness of parameter estimation in graphical models. In Advances in Neural Information Processing Systems (NeurIPS'14). 1062-1070.

[6] Guy Bresler, David Gamarnik, and Devavrat Shah. 2014. Structure learning of antiferromagnetic Ising models. In Advances in Neural Information Processing Systems (NeurIPS'14). 2852-2860.

[7] Guy Bresler and Mina Karzand. 2016. Learning a tree-structured Ising model in order to make predictions. arXiv:1604.06749.

[8] Guy Bresler, Elchanan Mossel, and Allan Sly. 2013. Reconstruction of Markov random fields from samples: Some observations and algorithms. SIAM fournal on Computing 42, 2 (2013), 563-578.

[9] Graham R. Brightwell and Peter Winkler. 2002. Random colorings of a Cayley tree. Contemporary Combinatorics 10 (2002), 247-276.

[10] Andrei A. Bulatov. 2005. H-coloring dichotomy revisited. Theoretical Computer Science 349, 1 (2005), 31-39. 
[11] C. K. Chow and C. N. Liu. 1968. Approximating discrete probability distributions with dependence trees. IEEE Transactions on Information Theory 14, 3 (1968), 462-467.

[12] Imre Csiszár and Zsolt Talata. 2006. Consistent estimation of the basic neighborhood of Markov random fields. Annals of Statistics 34, 1 (2006), 123-145.

[13] Sanjoy Dasgupta. 1999. Learning polytrees. In Proceedings of the 15th Conference on Uncertainty in Artificial Intelligence (UAI'99). 134-141.

[14] Amir Dembo, Andrea Montanari, and Nike Sun. 2013. Factor models on locally tree-like graphs. Annals of Probability 41, 6 (2013), 4162-4213.

[15] R. L. Dobrushin. 1968. The description of a random field by means of conditional probabilities and conditions of its regularity. Theory of Probability \& Its Applications 13, 2 (1968), 197-224.

[16] R. L. Dobrushin and S. B. Shlosman. 1985. Constructive criterion for the uniqueness of Gibbs field. In Statistical Physics and Dynamical Systems. Vol. 10. Birkhäuser Boston, Boston, MA, 347-370.

[17] Martin Dyer, Leslie Ann Goldberg, and Mark Jerrum. 2004. Counting and sampling $H$-colourings. Information and Computation 189, 1 (2004), 1-16.

[18] Martin Dyer, Leslie Ann Goldberg, and Mark Jerrum. 2008. Dobrushin conditions and systematic scan. Combinatorics, Probability and Computing 17, 6 (2008), 761-779.

[19] Martin Dyer and Catherine Greenhill. 2000. The complexity of counting graph homomorphisms. Random Structures and Algorithms 17, 3-4 (2000), 260-289.

[20] Martin Dyer, Alistair Sinclair, Eric Vigoda, and Dror Weitz. 2004. Mixing in time and space for lattice spin systems: A combinatorial view. Random Structures \& Algorithms 24, 4 (2004), 461-479.

[21] Nathan Eagle, Alex Sandy Pentland, and David Lazer. 2009. Inferring friendship network structure by using mobile phone data. Proceedings of the National Academy of Sciences 106, 36 (2009), 15274-15278.

[22] Thomas Emden-Weinert, Stefan Hougardy, and Bernd Kreuter. 1998. Uniquely colourable graphs and the hardness of colouring graphs of large girth. Combinatorics, Probability and Computing 7, 4 (1998), 375-386.

[23] Andreas Galanis, Leslie Ann Goldberg, and Mark Jerrum. 2016. Approximately counting $H$-colorings is \#BIS-hard. SIAM Journal on Computing 45, 3 (2016), 680-711.

[24] Andreas Galanis, Daniel Štefankovič, and Eric Vigoda. 2015. Inapproximability for antiferromagnetic spin systems in the tree non-uniqueness region. Journal of the ACM 62, 6 (2015), 50.

[25] Hans-Otto Georgii. 2011. Gibbs Measures and Phase Transitions. In De Gruyter Studies in Mathematics, Vol. 9. Walter de Gruyter, Boston, MA.

[26] Leslie Ann Goldberg, Steven Kelk, and Mike Paterson. 2002. The complexity of choosing an $H$-colouring (nearly) uniformly at random. In Proceedings of the 34th Annual ACM Symposium on Theory of Computing (STOC'02). ACM, New York, NY, 53-62.

[27] Linus Hamilton, Frederic Koehler, and Ankur Moitra. 2017. Information theoretic properties of Markov random fields, and their algorithmic applications. In Advances in Neural Information Processing Systems (NeurIPS'17). 2460-2469.

[28] Pavol Hell and Jaroslav Nešetřil. 1990. On the complexity of $H$-coloring. fournal of Combinatorial Theory, Series B 48, 1 (1990), 92-110.

[29] Pavol Hell and Jaroslav Nešetřil. 2004. Graphs and Homomorphisms. Oxford Lecture Series in Mathematics and Its Applications. Oxford University Press

[30] John P. Huelsenbeck, Fredrik Ronquist, Rasmus Nielsen, and Jonathan P. Bollback. 2001. Bayesian inference of phylogeny and its impact on evolutionary biology. Science 294, 5550 (2001), 2310-2314.

[31] Ali Jalali, Pradeep Ravikumar, Vishvas Vasuki, and Sujay Sanghavi. 2011. On learning discrete graphical models using group-sparse regularization. In Proceedings of the 14th International Conference on Artificial Intelligence and Statistics. 378-387.

[32] Johan Jonasson. 2002. Uniqueness of uniform random colorings of regular trees. Statistics \& Probability Letters 57, 3 (2002), 243-248.

[33] Adam Klivans and Raghu Meka. 2017. Learning graphical models using multiplicative weights. In Proceedings of the 58th Annual Symposium on Foundations of Computer Science (FOCS'17). IEEE, Los Alamitos, CA, 343-354.

[34] Su-In Lee, Varun Ganapathi, and Daphne Koller. 2007. Efficient structure learning of Markov networks using $L_{1}$ regularization. In Advances in Neural Information Processing Systems (NeurIPS'07). 817-824.

[35] Liang Li, Pinyan Lu, and Yitong Yin. 2013. Correlation decay up to uniqueness in spin systems. In Proceedings of the 24th Annual ACM-SIAM Symposium on Discrete Algorithms (SODA'13). 67-84.

[36] Daniel Marbach, James C. Costello, Robert Küffner, Nicci Vega, Robert J. Prill, Diogo M. Camacho, Kyle R. Allison, Manolis Kellis, James J. Collins, and Gustavo Stolovitzky. 2012. Wisdom of crowds for robust gene network inference. Nature Methods 9, 8 (2012), 796-804.

[37] Fabio Martinelli, Alistair Sinclair, and Dror Weitz. 2007. Fast mixing for independent sets, colorings, and other models on trees. Random Structures \& Algorithms 31, 2 (2007), 134-172. 
[38] Michael Molloy and Bruce Reed. 2001. Colouring graphs when the number of colours is nearly the maximum degree. In Proceedings of the 33rd Annual ACM Symposium on Theory of Computing (STOC'01). ACM, New York, NY, 462-470.

[39] Faruck Morcos, Andrea Pagnani, Bryan Lunt, Arianna Bertolino, Debora S. Marks, Chris Sander, Riccardo Zecchina, José N. Onuchic, Terence Hwa, and Martin Weigt. 2011. Direct-coupling analysis of residue coevolution captures native contacts across many protein families. Proceedings of the National Academy of Sciences 108, 49 (2011), E1293E1301.

[40] Pradeep Ravikumar, Martin J. Wainwright, and John D. Lafferty. 2010. High-dimensional Ising model selection using $\ell_{1}$-regularized logistic regression. Annals of Statistics 38, 3 (2010), 1287-1319.

[41] Stefan Roth and Michael J. Black. 2005. Fields of experts: A framework for learning image priors. In Proceedings of the 2005 IEEE Computer Society Conference on Computer Vision and Pattern Recognition (CVPR'05), Vol. 2. IEEE, Los Alamitos, CA, 860-867.

[42] Elad Schneidman, Michael J. Berry II, Ronen Segev, and William Bialek. 2006. Weak pairwise correlations imply strongly correlated network states in a neural population. Nature 440, 7087 (2006), 1007.

[43] Mark H. Siggers. 2010. A new proof of the $H$-coloring dichotomy. SIAM fournal on Discrete Mathematics 23, 4 (2010), 2204-2210.

[44] Allan Sly. 2010. Computational transition at the uniqueness threshold. In Proceedings of the 51st Annual Symposium on Foundations of Computer Science (FOCS'10). IEEE, Los Alamitos, CA, 287-296.

[45] Allan Sly and Nike Sun. 2012. The computational hardness of counting in two-spin models on $d$-regular graphs. In Proceedings of the 53rd Annual Symposium on Foundations of Computer Science (FOCS'12). IEEE, Los Alamitos, CA, 361-369.

[46] Nathan Srebro. 2001. Maximum likelihood bounded tree-width Markov networks. In Proceedings of the 17th Conference on Uncertainty in Artificial Intelligence (UAI'01). 504-511.

[47] Marc Vuffray, Sidhant Misra, Andrey Lokhov, and Michael Chertkov. 2016. Interaction screening: Efficient and sample-optimal learning of Ising models. In Advances in Neural Information Processing Systems (NeurIPS'16). 25952603.

[48] Dror Weitz. 2005. Combinatorial criteria for uniqueness of Gibbs measures. Random Structures \& Algorithms 27, 4 (2005), 445-475.

[49] Dror Weitz. 2006. Counting independent sets up to the tree threshold. In Proceedings of the 38th Annual ACM Symposium on Theory of Computing (STOC'06). ACM, New York, NY, 140-149.

Received May 2018; revised August 2019; accepted February 2020 